\title{
1. Horizontal connectivity in V1 : Prediction of coherence in contour and motion integration
}

2.

3. Abbreviated Title : Horizontal propagation of "network belief" in V1

4.

5. Benoit Le Bec ${ }^{19}$, Xoana G. Troncoso ${ }^{1,2 \&}$, Christophe Desbois ${ }^{1,3 \&}$, Yannick Passarelli ${ }^{1}$,

6. Pierre Baudot ${ }^{1,4}$, Cyril Monier $^{1}$, Marc Pananceau ${ }^{1 \&}$ and Yves Frégnac ${ }^{1 *}$.

7.

8. Place of work and Affiliations :

9. ${ }^{1}$ : NeuroPSI-UNIC, Institute of Neuroscience, CNRS, Paris Saclay University, France.

10. ${ }^{2}$ : Coaffiliation: Ecole Nationale Vétérinaire d'Alfort, Maisons-Alfort, France.

11. ${ }^{3}$ : Present address: Institut Polytechnique, Palaiseau, France.

12. ${ }^{4}$ : Present address: Median Technologies, Sophia-Antipolis, France.

13.

14. *: Corresponding Author:

15. E-mail : yves.fregnac@cnrs.fr (YF)

16. 


\section{Abstract}

19. This study demonstrates the functional importance of the Surround context relayed laterally in V1 by

20. the horizontal connectivity, in controlling the latency and the gain of the cortical response to the

21. feedforward visual drive. We report here four main findings : 1) a centripetal apparent motion

22. sequence results in a shortening of the spiking latency of V1 cells, when the orientation of the

23. local inducer and the global motion axis are both co-aligned with the RF orientation preference; 2) this

24. contextual effects grows with visual flow speed, peaking at $150-250^{\circ} / \mathrm{s}$ until matching the propagation

25. speed of horizontal connectivity $(0.15-0.25 \mathrm{~mm} / \mathrm{ms})$; 3) For this speed range, axial sensitivity of $\mathrm{V} 1$

26. cells is tilted by $90^{\circ}$ to become co-aligned with the orientation preference axis; 4) the modulation

27. strength by the surround context correlates with the spatiotemporal coherence of the apparent

28. motion flow. Our results suggest an internally-generated binding process, linking local (orientation

29. /position) and global (motion/direction) features as early as V1. This long-range diffusion process

30. constitutes a plausible substrate in $\mathrm{V} 1$ of the human psychophysical bias in speed estimate for

31. collinear motion. Since demonstrated in the anesthetized cat, this novel form of contextual control of

32. the cortical transfer function is a built-in property in V1, whose expression does not require behavioral

33. attention and top-down control from higher cortical areas. We propose that horizontal connectivity

34. participates to the propagation of an internal "prediction" wave, linking contour co-alignment and

35. global axial motion at an apparent speed in the range of saccadic-like eye-movements.

36. 


\section{Introduction}

38. Low-level visual perception is often described as a "pop-out" holistic process, elevated at the

39. state of "consciousness", independently of behaviorally directed attention. At the perceptual level,

40. Gestalt rules link stimuli according to feature similarity, spatial proximity or common fate $(1,2)$. Their

41. neural implementation is thought to occur at various stages of visual cortical processing (3) and best

42. captured, in terms of receptive field (RF) computation, as the combination of a smooth spatial

43. "association field" operator in space $(4,5)$, and a diffusion kernel in time (6). However, the detailed

44. synaptic implementation and the cortical regionalization of this dual space-time mechanism remain

45. largely unknown (review in (7)). An unsolved issue, which is central to this study, is to determine to

46. which degree "horizontal" connections, intrinsic to the primary visual cortex (V1), are instrumental to

47. the neural implementation of Gestalt laws.

48. Classically, the consensus is that binding in space and time are processed in separate cortical

49. areas, respectively in V1 for local attributes of form and contour integration, and in MT for global

50. motion (8-10). Although the functional expression of contour-related responses in $\mathrm{V} 1$ has been shown

51. to depend on the behavioral attention context and to be task-specific (11), it requires the triggering of

52. a stimulus-driven bottom-up process already detectable in the anesthetized brain (12-14). Local

53. circuits, intrinsic to primary visual cortex such as the long-range horizontal connections, are involved in

54. contour integration, but the final binding process is gated and tuned in strength by selective top-down

55. signals conveyed by re-entrant cortico-cortical pathways $((11,15)$; review in $(16))$. In contrast, in terms

56. of hypothetical cortical circuitry contribution, neural correlates of motion binding appear well

57. established in MT (17-19) but far less documented in V1 (but see (20)).

58. In spite of many intracellular receptive field (RF) studies in cat V1 (21-24), only a few have directly

59. measured the synaptic nature of contextual influence originating from the "silent surround" (25-30). A

60. striking feature from our own observations is that the strength of distal synaptic echoes exponentially

61. decreases, while their latency linearly increases, with the relative eccentricity of the stimulus from the 
62. RF center. If intracellular studies quantify synaptic convergence of lateral input, optical imaging, in a

63. converse way, monitors the full network depolarization pattern and dynamics in response to a local

64. stimulus. Intrinsic imaging reveals the spatial extent of the diffusive pattern of intracortical activity,

65. spread across several hypercolumns (up to $6^{\circ}$ in cat, in (31)). Mesoscopic dynamic imaging, using

66. voltage sensitive dyes with a temporal activation precision in the ms range, report depolarizing front

67. waves propagating across the superficial layers of $\mathrm{V} 1$, observed both in the ongoing and visually driven

68. states (non human primate (NHP) (32-36); cat (37-40); rat (41)). Their speed range is comparable

69. across mammalian species and matches remarkably that inferred from our intracellular recordings in

70. the cat (between 0.05 to $0.50 \mathrm{~mm} / \mathrm{ms}$ in (26)).

71. Intracellular recordings provide a unique way to dissect out the diversity of synaptic input

72. connectivity pattern across individual V1 cells and, at the same time, infer, through selective

73. averaging, the mean functional kernels of feedforward and lateral synaptic inputs composing the V1

74. receptive field. A prior study in our lab (29) proposed a new feature-specific averaging method, while

75. keeping the mapping stimulus geometry invariant, across cells, in a receptive-field-centric framework.

76. This was done by realigning each of the individual subthreshold maps relatively to a common origin

77. and " $0^{\circ}$ " reference axis, corresponding respectively to the center and the preferred

78. orientation/direction of each recorded RF. The averaging of 25 cells demonstrated that, irrespectively

79. of single cell RF diversity, the mean synaptic connectivity kernel of V1 neurons receives predominantly

80. iso-oriented inputs from neighboring collinear V1 hypercolumns, and that its spatial organization

81. pattern exactly mirrors the perceptual "Association Field" for collinear contours in human

82. psychophysics $(4,42)$.

83. The extensive intracellular study on dynamic Center-Surround interaction we present here - on a

84. larger cell sample $(n=65)$ - is based on the same premise. It searches to maximize the paired neighbor-

85. to-neighbor interactions between Surround and Center and quantify a novel form of contextual

86. control on the spiking responsiveness of V1 cells. For this purpose, we have designed here a variety of

87. multi-stroke apparent motion (AM) sequences of specific speed and spatial anisotropy, which, under 
bioRxiv preprint doi: https://doi.org/10.1101/2021.09.10.459746; this version posted September 11, 2021. The copyright holder for this preprint (which was not certified by peer review) is the author/funder. All rights reserved. No reuse allowed without permission.

Horizontal propagation of "network belief" in V1

20 August 2021

88. precise timing and local feature co-alignment conditions, allowed us to control the temporal phase

89. between horizontal and feedforward inputs. Two sets of visual protocols were applied separately in

90. two distinct sets of cells with complementary objectives. The "cardinal" protocol (Fig. 1B), was

91. designed to : i) optimize the visibility of synaptic inputs originating laterally from the "Near" RF

92. surround, outside the spiking "minimal discharge field" (MDF), but potentially overlapping with the

93. subthreshold depolarizing receptive field (SRF)); ii) probe for a possible axial bias in the preferred

94. recruitment of contextual Surround information integration, using 2-3 stroke AM sequences along the

95. two main (length/width) axis of the RF). The second series of protocols, termed "radial" (Fig. 1C), was

96. based on a more conservative definition of the synaptic feedforward cortical imprint, no longer

97. defined only by its spiking discharge field (MDF) but including as well the surrounding depolarizing SRF.

98. This additional requirement limited the Surround stimulation to "Far" regions outside the SRF only. It

99. was meant to minimize the risk of spurious feedforward contamination when probing the location the

100. closest to the RF borders (position D1 in Fig. 1A). To compensate for potentially weaker Surround

101. responses, we increased the number of AM strokes and imposed radial symmetry in order to recruit

102. simultaneously more sources in the RF periphery at the same relative Surround eccentricity.

103.

104. Fig 1. Working Hypothesis and Visual Stimulation Protocols

105. [A] : Working Hypothesis: the AM sequence is designed such as to synchronize the synaptic activation time of

106. horizontal volleys, evoked by the sequential presentation of 2 to 5 Gabor patches (GP) in the Surround, with the

107. feedforward activation of the RF Center (shaded rectangle in the D0 position). The Surround GPs, regularly

108. spaced from "Far" (D5) to "Near" (D1) periphery along the apparent motion path (horizontal axis), are flashed in

109. succession at high contrast. The test reference GP, terminating the AM sequence, is flashed at low/high contrast

110. in the RF center. Each orange arrow represents the lateral propagation of the presynaptic volley elicited by each

111. distal GP. Other synaptic recruitment paths potentially contribute also to Surround-Center modulation, in

112. particular cascades of proximal neighbor-to-neighbor links (grey arrows).

113. [B] "Cardinal" Protocol : the two columns illustrate the exploration of the two cardinal axis (horizontal for the

114. RF orientation preference; vertical for the RF width axis) with 2 or 3-stroke AM sequences of iso-oriented stimuli 
bioRxiv preprint doi: https://doi.org/10.1101/2021.09.10.459746; this version posted September 11, 2021. The copyright holder for this preprint (which was not certified by peer review) is the author/funder. All rights reserved. No reuse allowed without permission.

Horizontal propagation of "network belief" in V1

115. ("collinear", first row; "parallel", second row) or cross-oriented stimuli (two bottom rows, same motion paths

116. but the local inducer is now orthogonal to the RF orientation preference).

117. [C]: "Radial" Protocol : the two columns illustrate the various AM flow patterns (individual rows), either

118. funneled along a given motion axis (SECTOR, left), radially contracting (CP: centripetal) or expanding (CF:

119. centrifugal), or across the full Surround (FULL, right). In the SECTOR condition, two opposite motion axes are

120. symmetrically explored, either aligned with the RF orientation preference (horizontal axis $\left( \pm 30^{\circ}\right)$ in rows 1,2 and

121. 4) or its width axis (vertical axis $\left( \pm 30^{\circ}\right)$, row 3$)$. In the FULL condition, all directional motion axes (discretized by

122. $30^{\circ}$ steps) are stimulated concomitantly and intersect the RF center (horizontal grey icon). From top to bottom,

123. each row illustrates one specific configuration of AM flow: 1) centripetal collinear flow (CP-ISO) from Surround to

124. Center (red, row 1); 2) centrifugal collinear flow (CF-ISO) from Center to Surround (green, row 2), 3) centripetal

125. cross-oriented flow (CP-CROSS) across the RF width axis (gold, row 3); 4) randomized collinear pattern (RND-ISO)

126. where each successive GP location has been randomized in space and time (blue, bottom row). Note that in all

127. the conditions other than CF-ISO, the last GP of the sequence is flashed in the RF center. See Text for details.

128.

129. The "working hypothesis" we tested posits that the spatio-temporal optimization of the cortical

130. responsiveness control should be reached, where, in space, the local orientation of each Surround GP

131. matches that of the recorded RF (as expected from the "Association Field" profile), and when, in time,

132. the phase of the stimulation of each surround node compensates for the lag of the postsynaptic

133. response, due to the dependency of the PSP latency on the eccentricity relative to the RF center

134. (Fig. 1A, "latency basin" in Fig. 2A). Our functional prediction is that a latency shortening and an

135. overall cortical gain amplification of the composite PSP produced by the AM sequence invading the RF

136. center should be observed when the global flow speed is adjusted to merge in synchrony the

137. respective synaptic impacts of the lateral and feedforward drives onto the intracellularly recorded cell.

138. This study addresses the importance of the Surround context dynamics relayed laterally in V1 by

139. the horizontal connectivity, in controlling the timing and the responsiveness gain of the cortical

140. response. It also gives evidence for neural correlates of "filling-in" responses at the earliest stage of

141. visual cortical processing, i.e. the primary visual cortex. Remarkably, these responses are shown to be 
bioRxiv preprint doi: https://doi.org/10.1101/2021.09.10.459746; this version posted September 11, 2021. The copyright holder for this preprint (which was not certified by peer review) is the author/funder. All rights reserved. No reuse allowed without permission.

Horizontal propagation of "network belief" in V1

20 August 2021

142. specific of co-alignment, and their timing anticipates the occurrence of the same orientation feature

143. ("expected" in Bayesian terms) in the RF center, even in the absence of feedforward activation. We

144. infer, from a detailed contextual synaptic interaction analysis, a conceptual framework which accounts

145. for the neural correlates in V1 of specific Gestalt-like visual configurations, linking local (orientation)

146. and global (contour integration, motion) features.

147. 


\section{Material and Methods}

\section{1. Animal preparation and electrophysiological recordings}

150. All experiments were performed in anesthetized (alfaxalone, Alfaxan ${ }^{\circledR}, 2.5 \mathrm{mg} / \mathrm{kg} / \mathrm{hr}$ ) and

151. paralyzed (pancuronium Pavulon ${ }^{\circledR}\left(0.1 \mathrm{ml} / \mathrm{kg} / \mathrm{hr}\right.$ ) or rocuronium Esmeron ${ }^{\circledR}, 4 \mathrm{mg} / \mathrm{kg} / \mathrm{hr}$ ) adult cats of

152. either sex, in accordance with the American Physiological Society's Guiding Principles for the Care and

153. Use of animals. Animals were bred in the Central CNRS Animal Care Facilities under veterinary and

154. National Ethical Committee supervision (MESR agreement: $n^{\circ} 201603111-3088501$ ). Anesthesia depth,

155. analgesia level and the general physiological state stability were continuously monitored from the

156. recording and analysis of EKG and extradural ECoG signals. End-tidal CO2 was maintained between 3.5

157. and $4.5 \%$, and body temperature was regulated around $38^{\circ} \mathrm{C}$ throughout the experiment. Once the

158. animal's head was fixed to the stereotaxic frame, and the craniotomy performed, we checked again

159. the presence of a slit pupillary state, indicative of adequate anesthesia during the surgery phase. Then,

160. atropine (1\%) and phenylephrine (5\%) were applied to both eyes to dilate pupils, block the

161. accommodation and withdraw nictitating membranes, while artificial pupils and corrective lenses

162. were used to protect the eyes and focus the backprojection of the eye fundus on the stimulation

163. screen.

164. In vivo intracellular recordings of cortical cells were performed in the primary visual cortex (area

165. 17) using sharp Glass electrodes pulled from 1.5-mm-thick borosilicate capillaries (World Precision

166. Instruments Inc.). Electrodes were filled with a $2 \mathrm{M}$ potassium methyl sulfate ( $\mathrm{pH}$ ranging from 7.2 to

167. 7.4), $4 \mathrm{mM} \mathrm{KCl}$ solution for compensating the tip potential, and $1.5 \%$ biocytin. Electrode resistances

168. before impalement ranged between 70 and $130 \mathrm{M} \Omega$. Cells were recorded in current clamp bridge

169. mode using an Axoclamp 2A (Axon Instrument inc.) or a SEC-05X (NPI inc.). The average resting

170. potential was between -62 to $-78 \mathrm{mV}$ (mean $\pm \sigma=-66,8 \pm 8,3 \mathrm{mV}$ ) with no or a slight holding current

171. (<= $<-0.5 \mathrm{nA}$ ) when needed. Signals were filtered and amplified with a CyberAmp380 (Axon Instrument

172. inc.) and digitized at $10 \mathrm{kHz}$ by an ITC-1600 (InstruTECH ${ }^{\circledR}$ ) card. Based on recording depth and the 
173. laminar location and morphological identification of fourteen biocytin labeled cells, the overall

174. sampling was distributed from supra- to infra-granular layers. However, in view of the limited number

175. of labelled cells (experimentally constrained by gliolysis increase with the length of recording) and a

176. slight bias for recordings in deep layers, no laminar study was done in this reduced sample.

177.

\section{2. Visual stimulation}

179. Visual stimuli were generated, using the ELPHY home-made software (G. Sadoc, UNIC-CNRS), on a

180. CRT P31 green monochrome monitor positioned at $57 \mathrm{~cm}\left(1 \mathrm{~cm}=1^{\circ}\right.$ of visual angle) at refresh rate of

181. $60 \mathrm{~Hz}$ (19 inches, $640 \times 480$ pixels) for the "cardinal" protocols, and on a gamma-corrected LCD green

182. monitor at $28.5 \mathrm{~cm}$ from the cat's retinal plan $\left(0.5 \mathrm{~cm}=1^{\circ}\right.$ of visual angle $)$ at a refresh rate of $144 \mathrm{~Hz}$

183. (27 inches screen, $1920 \times 1080$ pixels) for the "radial" protocols.

\section{2.1. Initial quantification of RF optimal features :}

185. The receptive field (RF) position of each recorded neuron and its ocular dominance were initially

186. established using moving light bars. Stimulation was done through the dominant eye for the rest of the

187. experiment. Subthreshold ON and OFF (SRF) sub-regions and spike minimal discharge fields (MDF)

188. were then mapped by forward correlation of visual responses to a random "sparse noise" (SN)

189. protocol where white $\left(40 \mathrm{~cd} / \mathrm{m}^{2}\right)$ and dark $\left(1 \mathrm{~cd} / \mathrm{m}^{2}\right)$ non-overlapping squares were presented one at a

190. time for $16-34 \mathrm{~ms}$ against a grey background $\left(20 \mathrm{~cd} / \mathrm{m}^{2}\right)$ in each position of the exploration grid ( $8 \times 8$ or

191. 20x20 pixels) centered over the coarse RF region. In some cells, an additional 1D mapping was done

192. with an optimally oriented thin long bar flashed randomly at various eccentricities across the RF

193. width (Fig. 2A).

194. Once the RF location defined, the preferred orientation, phase and spatial frequency of the cell

195. were determined using a dense Gabor Noise (GN) stimulation protocol (43), in which flashed (16-34

196. ms) Gabor patches (GPs, adjusted in size to the s-DF extent) were randomly presented at six different

197. orientations (with $30^{\circ}$ steps), four phases $\left(0^{\circ}, 90^{\circ}, 180^{\circ}\right.$ and $270^{\circ}$ ) and five spatial periods (ranging 
bioRxiv preprint doi: https://doi.org/10.1101/2021.09.10.459746; this version posted September 11, 2021. The copyright holder for this preprint (which was not certified by peer review) is the author/funder. All rights reserved. No reuse allowed without permission.

Horizontal propagation of "network belief" in V1

20 August 2021

198. from 0.1 to 4.9 cycles per degree of visual angle). For each cell, the parameters of the Gabor patch

199. eliciting the strongest response in the RF center were retained to compose a template of preferred

200. features of the inducer (independently of its position) in the subsequent apparent motion (AM)

201. sequences. In order to evoke a sizeable synaptic "horizontal" response, the GP flashed in the Surround

202. were always presented at high contrast (mean $\pm \sigma=92.5 \pm 11.5 \%$; range $75-100 \%$ ), whereas the

203. feedforward central stimulus was flashed in the MDF either at low or medium contrast (mean \pm

204. $\sigma=40,6 \pm 24.5 \%$; range $(25-100 \%))$.

\section{2.2. "Cardinal" Apparent Motion (AM) Protocol :}

206. In order to assess the dependency of the center-surround interaction on local orientation and

207. global motion, we first designed three-stroke center-surround AM protocols for which the Gabor

208. patches were flashed along the two "cardinal" RF axes defined by the RF preferred orientation and

209. width (Fig. 1B). We looked at the modulatory effect that a two-stroke activation of the "silent"

210. surround would produce (along each of these axis) on the response to a test stimulus flashed within

211. the MDF (the third stroke). Randomly interleaved three-stroke stimulation sequences were applied

212. moving away from the receptive field center ("centrifugal" (CF) condition, green arrows in Fig. 1B), or

213. from the surround to the center ("centripetal" (CP) condition, red arrows) from one side of the RF at a

214. time.

215. The size of the Gabor patch mask was set at $150 \%$ of the length of the spiking discharge field

216. while the standard deviation of its Gaussian envelope was set at $20 \%$. The stimulus inter-node distance

217. was of $120 \%$ of its length, often leading the more proximal GP to partially encroach on the border of

218. the impulse SRF. The orientation of the local inducer could be defined two ways, either in relation with

219. the RF orientation preference, or in relation with the AM motion axis. These two analysis contexts will

220. be considered separately in the Results. Here, by sake of uniformity with the "radial" protocol, the

221. ISO- and CROSS- conditions are defined relative to the chosen AM axis. The complete set of 16

222. Surround-Only stimulations (Fig. 1B, left column) included 8 conditions [CP-ISO_main-axis, CP-

223. ISO_width-axis, CP-CROSS_main-axis, CP-CROSS_width-axis, CF-ISO_main-axis, CF-ISO_width-axis, CF- 
224. CROSS_main axis, CF-CROSS_width axis] X 2 direction conditions (one side at a time)], to which 2

225. control conditions (1 Center-Only +1 Blank trial, where no stimulus was presented) were added.

226. Hence a total of 18 conditions was completed in 13 cells. Center-Surround interactions were tested in

227. 23 cells with full AM sequences terminating in the RF center (Fig. 1B, right column), under 10

228. conditions [4 conditions (CP-ISO_main-axis, CP-CROSS_width-axis, CF-ISO_main-axis, CF-CROSS_width-

229. axis) $\times 2$ directions (one side at a time) + Center-Only stimulation +1 blank]. For each study

230. (Surround-Only; Surround-Center), the different stimulus conditions were interleaved randomly in

231. each trial block. Recordings lasted for 20 blocks.

232. Given the constraint of the monitor distance $(57 \mathrm{cms})$, its refreshing rate $(60 \mathrm{~Hz})$, and the fixed

233. duration of the static Gabor inducer presentation (16 ms), individual cell AM speed values only

234. depended on the size of the recorded RF which was used to define the sequential interstimulus step

235. distance. Accordingly, AM speeds were preset in the suitable range of values to compensate for the

236. dependency of visual PSP latency with relative eccentricity $(26,29)$.

\section{2.3. "Radial” Apparent Motion (AM) Protocol :}

238. This series of protocols was done with a larger (27 inches) and faster screen (144 Hz), using a

239. shortened viewing distance $(28.5 \mathrm{cms})$, which allowed a more extensive exploration of the "Far"

240. surround. The visual field was paved with a grid composed of 5 concentric rings of increasing

241. eccentricity centered on the RF covering up to $25^{\circ}$ in the surround (Fig. $1 \mathrm{C}$ ). To the difference of the

242. "cardinal" protocol, where stimulus size and spacing were defined relatively to the spiking MDF size,

243. the distance between each outer ring and the patch size were adjusted in this new protocol to the full

244. extent of the subthreshold depolarizing receptive field (SRF) mapped with impulse stimuli. This change

245. in the RF core definition, from that used in the "cardinal" protocol condition, was done to prevent, on

246. a cell-by-cell basis, a direct feedforward contamination by the stimulation of the most proximal

247. Surround location (position D1 in Fig. 1A).

248. Additionally, in the "radial" protocol, we designed Apparent Motion sequences whose spatio- 
249. temporal coherence was parametrized to dissect the dependency of the inducer orientation relative to

250. the motion path, as well as the spatio-temporal congruency within the AM flow (see Results). For each

251. recorded cell, the reference AM sequence was the centripetal iso-oriented configuration (CP-ISO, top

252. "red" row in Fig. 1C), where the local inducer orientation was either co-aligned along the preferred

253. orientation axis of the recorded RF (SECTOR configuration, left column in panel 1C) or along the global

254. motion axes (FULL configuration, right column). Three supplementary visual AM configurations were

255. tested, in which the spatio-temporal structure of the local Gabor patch (GP, local inducer) sequence

256. was altered, while keeping the overall stimulus energy distribution unchanged: i) in the first control

257. condition, namely the centrifugal iso-oriented AM sequence (CF-ISO, second "green" row from the

258. top, Fig. 1C), the temporal order of the sequential presentation of the co-aligned GPs was reversed,

259. resulting in a centrifugal (CF) flow from Center to "Far" Surround locations; ii) the second control was

260. designed to study the contextual impact of the local GP orientation relative to the motion axis. In the

261. centripetal cross-oriented AM condition, the local orientation of the inducer was orthogonal to the

262. motion axis (CP-CROSS, third "yellow" row from the top, Fig. 1C); iii) in the third control condition, the

263. same retinal space (SECTOR or FULL) as in the CP-ISO condition of interest was stimulated with the

264. same GPs (i.e., with the same number, features, and energy distribution at each step of the AM) but

265. the coherence of the flows was randomized both in space and time (RND-ISO, bottom "blue" row). For

266. each block, each stimulus location was visited only once, and the last stimulus location was the RF

267. center. The repeated sequences were identical across blocks (pseudo-randomization).

268. The stimulus set in the "radial" protocol - where all contextual conditions were explored - was

269. composed of 16 dynamic apparent motion sequences ( 4 conditions (CP-ISO, CF-ISO, CP-CROSS, RND-

270. ISO) $\times 2$ trajectories (with or without Center stimulation) $\times 2$ configurations (SECTOR and FULL), and 21

271. stimulation types where Gabor Patches were flashed in isolation (5 peripheral locations $X 2$ conditions

272. X 2 configurations $+1 \mathrm{GP}$ flashed in the Center). In each block of trials, each stimulus was presented

273. only once. Additionally, 6 "blank" trials were also included in each block. The resulting set of 43

274. stimulations was presented in random order in each block. Recordings lasted for 19-50 blocks (median 
275. 50).

276. In order to optimize synaptic summation between Surround and Center stimulation, the AM

277. sequence speed was function of the spatial extent of the SRF and of the stroke duration of Gabor

278. patches and adjusted cell-by-cell in order to fit the latency basin of "Far" surround response latencies.

279. In the "radial" protocol where AM speed and stroke duration could be independently parametrized,

280. the stroke duration was in the $30 \mathrm{~ms}$ range ( $\pm 9 \mathrm{~ms}$ ). It was $16.6 \mathrm{~ms}$ in the "cardinal" protocol.

281.

\section{3. Intracellular Data analysis}

\section{3.1. Data formatting :}

284. Spikes were threshold-detected in the raw traces and replaced by alpha functions in the membrane

285. potential $(\mathrm{Vm})$ traces $\left(\alpha(t)=\mathrm{a}^{*} \mathrm{t}^{*} \exp (-\mathrm{t} / \tau)\right)$, where $\mathrm{a}$ is the slope at the peak of the second derivative of

286. the rising phase, and $\tau$ is the half-width of the spike), as done in previous studies (29). Resulting traces

287. were band-pass filtered $(0.1-300 \mathrm{~Hz})$ and peristimulus triggered waveform (PSTW) were averaged

288. across trials and conditions. Prior to any statistical analysis, all traces were down-sampled to $1 \mathrm{kHz}$ and

289. smoothed using a sliding average window of $7 \mathrm{~ms}$. For spikes, individual peristimulus time histograms

290. were smoothed using a Gaussian window ( $\sigma=3 \mathrm{~ms})$. For a given trial block containing all conditions,

291. the corresponding mean activity (average Vm and Spiking discharge rate) was subtracted from the raw 292. PSTWs and PSTHs.

\section{3.2. Response quantification and statistical significance :}

294. For the "cardinal" protocol, Z-test statistics (one tailed $p<0.05$ ) were applied to mean population

295. integral response values of the subthreshold response waveforms (dominantly depolarizing) compared

296. to resting state fluctuations. For the "radial" protocol, since a larger spectrum of stimulus conditions

297. was used, more extensive statistical analyses were done on the individual cell's responses, without or

298. with selection criteria. All significance tests fell into two categories: quantifying the significance of a

299. response to a stimulus or comparing data statistics between two conditions. To do the former, we 
bioRxiv preprint doi: https://doi.org/10.1101/2021.09.10.459746; this version posted September 11, 2021. The copyright holder for this preprint (which was not certified by peer review) is the author/funder. All rights reserved. No reuse allowed without permission.

Horizontal propagation of "network belief" in V1

20 August 2021

300. relied on the activity during blank trials, which provides the null distribution for visual responses (i.e.,

301. the activity that would occur should the cell not respond to the stimulus, which was our "null"

302. hypothesis). Consequently, for each cell, the average ongoing synaptic or spiking activity recorded

303. during "blank" trials was subtracted from each evoked PSTW/PSTH trial activity. For the latter, we

304. used cell-by-cell paired comparison between distinct tested conditions. Statistical analysis was then

305. done using a randomization permutation test with $10^{4}$ repetitions. In the remaining fifteen "non-

306. spiking" cells, analysis was restricted to subthreshold activity.

307. From these computations, we extracted the null hypothesis waveform envelope for a given

308. confidence interval at each point in time. Only cells with a response to the Center-Only condition

309. significantly larger than the blank $(p<0.01)$ in the interval 0 -to-120 ms (" 0 " being the onset of the last

310. stimulation in the center when the RF was actually stimulated, " 120 " ms integrating most of the

311. feedforward onset and offset responses) were considered for further analysis. For PSTW and PSTHs,

312. respectively thirty-seven and twenty-two cells were retained after this verification and constitute the

313. population used for the Results section for the "radial" protocol.

\section{3.3. Quantification of the contextual change in response gain and phase:}

315. We quantified the time-course and the strength of the contextual control of the cortical transfer

316. function by measuring the changes in i) subthreshold latency and ii) the integral value of the

317. depolarizing postsynaptic potential (PSP) response to the complete AM sequence terminating in the

318. recorded cell's receptive field. For the "cardinal" protocol, the latency measure was defined by the

319. onset of the mean PSP reaching significance (Z-test, $p<0.05)$. For the "radial" protocol, we chose to use

320. a more conservative measure to avoid spurious detections of fluctuations in the ongoing synaptic

321. noise. Accordingly, the latency change ( $\Delta$ latency) was measured at half-height of the Center-Only

322. response amplitude peak, separately for $\mathrm{Vm}$ and spikes. For the response gain ( $\Delta$ response), we

323. measured the change in the PSP depolarizing envelope integral (and, eventually, spiking integral),

324. relative to the Center-Only response, over the whole time-course of the test-response. Here, the

325. rationale was to integrate differences in evoked responses for all possible time relationships between 
326. feedforward and lateral input. The same statistical analysis (randomization test, $10^{4}$ repetitions;

327. $\mathrm{p}<0.05$ ) was repeated for PSTWs and PSTHs for both criteria. The paired-analysis was replicated across

328. conditions to measure statistical significance between the main condition of interest (CP-ISO) and the

329. other contextual conditions.

\section{3.4. Surround-Only responses:}

331. For the "cardinal” protocol, statistical significance was assessed using Z-test statistics on response

332. integrals $(p<0.05)$. For the "radial" protocol, we used a permutation test $\left(10^{4}\right.$ repetitions; $\left.p<0.01\right)$. Due

333. to the noisy nature and the weak amplitude of purely lateral responses, we only retained $\mathrm{Vm}$

334. waveforms for which the statistical significance threshold was trespassed for at least 15 consecutive

335. milliseconds in the interval 0-120 ms.

\section{3.5. Linear predictions and Dynamic non-linearities:}

337. For the "radial" protocol, we determined in each individual case whether the actual Surround-

338. Only responses to dynamic sequences were larger than (or equal to) the sum of the temporally shifted

339. responses to each GP flashed in isolation at each location (reproducing the spatial and temporal

340. ordering of a virtual AM sequence). This linear predictor was in a first step compared to the actual AM

341. dynamic sequences before they reached the RF Center, to probe for a nonlinearity between the

342. integration of elements flashed in isolation in the surround and the actual build-up of anticipatory

343. activity during AM sequences. Because GP flashed in isolation in the close vicinity of the SDF already

344. induce significant depolarizing responses (29), we considered Surround-Only synaptic waveforms as

345. significantly larger than their "static" linear predictor, if they crossed the upper limit of the confidence

346. interval for a minimum duration of 7 consecutive $\mathrm{ms}$ in the interval 0-120 ms (permutation test, $10^{4}$

347. repetitions, $\mathrm{p}<0.05)$. A second predictor was calculated by subtracting the Center-Only response from

348. the complete AM "Surround-then-Center" response. This two-term linear predictor was compared to

349. the observed "Surround-only" AM response to test for a non-linearity between the integration of

350. contextual information in the "Near" Surround and the feedforward drive. 


\section{3.6. Population analysis:}

352. In order to compare responses between cells, each individual flow pattern's specific geometry -

353. defined on a cell-by-cell basis according to the preferred orientation and direction of each cell - was

354. realigned on a common " 0 " reference axis (visualized by the semi-horizontal axis pointing to the right

355. in all Figures), regardless of the absolute orientation preference of each cell. No normalization of

356. responses was used in the "cardinal" protocol. In the "radial" protocol, the amplitude of the PSTWs

357. and PSTHs of each cell were first normalized to the peak of the Center-Only response. Before

358. averaging, PSTWs and PSTHs of all the cells were also realigned with respect to the onset latency of

359. their individual Center-Only response (respectively, subthreshold and spiking), which was defined as

360. the time of the first point (blue dot) of the average Center-Only response departing from the "blank"

361. response mean by more than $3 \sigma$. This allowed a realignment of all normalized cell-by cell profiles and

362. the calculus of the mean population response profile.

363. 


\section{Results}

365. This intracellular study (65 cells) of Center-Surround interactions in V1 of the anesthetized cat is

366. based on two distinct visual protocols, applied in two different sets of cells (see Methods). The first

367. protocol, termed "cardinal" (Fig. 1B), recruiting both "Near" and "Far" RF surround, was applied to a

368. first batch of 23 cells. The second series of protocols, termed "radial" (Fig. 1C), limiting the Surround

369. stimulation to "Far" regions outside the SRF only, was performed on a second batch of 42 cells.

370. Accordingly, response averages were done separately for the two visual stimulation protocols.

\section{1. Probing synaptic responses from the "silent" Surround}

\section{1.1. Spatial summation}

373. The "silent Surround" is classically defined as the region outside the minimal discharge field

374. (MDF), where impulse stimuli do not evoke a significant spiking response. However, the intracellular

375. mapping of synaptic responses with sparse or dense noise (SN or DN), devoid of spatial summation,

376. shows a broader subthreshold (non-spiking) receptive field (SRF) extending beyond the border of the

377. MDF, and often invading the opponent contrast discharge field, even in Simple cells $(43,44)$. This

378. defines a Near-Surround domain, connex to the MDF, where the contribution of purely lateral synaptic

379. input cannot be unambiguously disentangled from subthreshold feedforward activation (25).

380. The mapping of the minimal discharge field (MDF) with small light or dark squares $\left(0.2-0.9^{\circ}\right)$

381. showed on average a spiking core field of $1-3^{\circ}$ of visual angle. Overall, the size of the impulse

382. subthreshold depolarizing receptive field (SRF) of the cortical neurons recorded in this study ranged

383. from $2.5^{\circ}$ to $7.5^{\circ}\left(5,1 \pm 1,6^{\circ}\right)$, for eccentricities from the area centralis between $1.1^{\circ}$ and $8.3^{\circ}(4.0 \pm$

384. $\left.1.6^{\circ}\right)$. This initial mapping was used to define, in a conservative way in each cell, the retinotopic

385. imprint of the feedforward input, whether it produced a spike (MDF) or only a depolarizing

386. subthreshold response (SRF). 
bioRxiv preprint doi: https://doi.org/10.1101/2021.09.10.459746; this version posted September 11, 2021. The copyright holder for this preprint (which was not certified by peer review) is the author/funder. All rights reserved. No reuse allowed without permission.

Horizontal propagation of "network belief" in V1

20 August 2021

387. In contrast, elongated light bars ( $5-12^{\circ}$ length) flashed across the RF width axis, which increases

388. input spatial summation, elicited synaptic responses, in the same cell, originating much further away

389. from the RF center ( $7.1^{\circ}$ for bars against $2.5^{\circ}$ for $\mathrm{SN}$ in the example cell in Fig. $\left.2 \mathrm{~A}\right)$. Using annular

390. gratings at the optimal phase and orientation, with still larger spatial summation, our lab previously

391. demonstrated distal synaptic responses originating beyond $10^{\circ}$ of relative eccentricity (Fig. 2B). These

392. different observations have established that the recruitment efficacy of lateral connectivity evoking

393. Surround responses strongly depends on the level of spatial summation and are selective to the

394. orientation feature of the stimulus flashed in the distal periphery $(26,27,45)$.

395.

396. Fig 2. A synaptic view of visual V1 receptive fields

397. [A] : Latency basin of synaptic responses relative to the RF Center : Top left, spatial maps of the ON- and OFF-

398. Depolarizing Fields latencies using sparse noise (SN) mapping. Heat scale: color code for latency values (from 0

399. (black) to $200 \mathrm{~ms}$ (yellow)). Bottom left, 1D-mapping of ON- and OFF- PSP responses in the same cell, evoked by

400. long bars $\left(7.1^{\circ} \times 0.7^{\circ}\right)$ flashed at the optimal orientation and positioned at different eccentricities (in ordinates,

401. ranging from $-4^{\circ}$ (bottom) to $+6^{\circ}$ (top)) across the RF width axis. Thick boxes (left ordinates) designate the

402. positions which are in overlap with the SRF mapping using SN (above, top left). Red (ON) and blue (OFF) linear

403. regressions illustrate the 1D-latency basin profiles of Surround-Only subthreshold responses. The linear fits of

404. latency versus eccentricity give apparent horizontal speeds of propagation estimates of 0.18-0.38 mm/ms for

405. ON- (red, left) and OFF- (blue, right) responses.

406. [B] Dependency on spatial summation (adapted from (26)) : From top to bottom, phase-reversal (2 Hz)

407. responses to disk [1] and annular gratings [2 \& 3], for increasing inner border eccentricities from the

408. RF center ( $0^{\circ}$ in [1], $5.6^{\circ}$ in [2] and $10.3^{\circ}$ in [3]). Note the strength decay and the onset latency increase in

409. Surround-Only responses [2 \& 3] with eccentricity, reaching 32 ms for the "Far" Surround annulus.

410. [C] Apparent Speed of Horizontal Propagation (ASHP) distribution : Stacked data histograms from (26)

411. using long bars (1D-mapping, blue) or sparse noise (green). Note that most values extracted from latency

412. basin slopes range between 0.05 and $0.60 \mathrm{~mm} / \mathrm{ms}$.

413. 


\section{1.2. Latency dependency of Surround responses with relative eccentricity}

415. In addition to the spatial extent of the RF, a pivotal observation, on which our working hypothesis

416. is grounded, is that the further away from the MDF center, the latency of subthreshold surround

417. responses progressively increases in a linear fashion with relative eccentricity, while the onset

418. latency and the rising phase of those surround-responses depends on the spatial summation extent of

419. the stimulus used to probe visual responsiveness. Fig. 2A illustrates the spatio-temporal latency

420. maps of depolarizing and spiking responses of a V1 cell for sparse noise (top panel) and of synaptic

421. subthreshold PSPs for optimally oriented long bars flashed at different eccentricities across the RF

422. width axis (bottom panel). In this latter case, the 1D-plots of ON- and OFF-responses can be used to

423. extract the slopes of the latency gradients with eccentricity relative to the RF center. These measures

424. allow to infer estimates of the mean apparent speed of horizontal propagation (ASHP), ranging here

425. from 0.18 and $0.38 \mathrm{~mm} / \mathrm{ms}$. When increasing spatial summation by using optimally oriented and

426. phased annular gratings, the resulting latency shifts can increase to $20-50 \mathrm{~ms}$ for relative eccentricity

427. values as large as $11^{\circ}$. The measured propagation speed (and conversely the onset latency of surround

428. responses) for flashed long bars (blue histogram in Fig. 2C) appears similar with that obtained from

429. sparse noise mapping (SN, green histogram), apart from tail values reaching larger values for long bars

430. (ASHP mean $\pm \sigma:$ (for $\mathrm{SN}$, green) $0.21 \pm 0.16 \mathrm{~mm} / \mathrm{ms}$; (for bars, blue) $0.27 \pm 0.22 \mathrm{~mm} / \mathrm{ms}$ ).

\section{1.3. Apparent Motion Axial sensitivity and Orientation tuning}

432. Once the Gabor template optimizing the Center response was determined (see Methods), each RF

433. and/or its Surround were tested with 2-stroke (Surround-Only) or 3-stroke apparent motion (AM)

434. sequences along the cardinal RF axes (Fig. 1B). These "cardinals" axes, extending symmetrically

435. around the RF center, correspond, one, to the "main" orientation preference axis (represented

436. arbitrarily by the horizontal, in all the figures) and the other, to the RF width axis (vertical). For each of

437. these axes, the orientation of the local Gabor inducer stimuli could be either similar, "ISO-RF", or

438. orthogonal, "CROSS-RF" to that of the RF orientation preference axis. 
bioRxiv preprint doi: https://doi.org/10.1101/2021.09.10.459746; this version posted September 11, 2021. The copyright holder for this preprint (which was not certified by peer review) is the author/funder. All rights reserved. No reuse allowed without permission.

Horizontal propagation of "network belief" in V1

20 August 2021

439. Examples of Surround-Only AM responses of the "cardinal" protocol are illustrated in Fig. 3A,

440. whose magnitude can reach the size of the response to a low contrast input in the center (compare

441. Surround-Only CP-ISO AM responses (red waveform) along the preferred orientation axis with the

442. Center-Only response (black waveform)). As a reference, the center insert in Fig. 3A illustrates the

443. mapping of the SRF border (white contour) and the ON- and OFF-discharge fields (respectively, filled in

444. red and blue). The surround source locations of GPs used in the AM sequence partially encroach on

445. the SRF border, but not on the MDF. The AM evoked synaptic responses are much stronger than the

446. impulse SN response, as shown by the absence of depolarizing responses to light (lower dark red

447. trace) and dark (blue trace) pixel stimulations, presented in the center of the lesser eccentricity

448. peripheral Gabor location. In this example, as in most recorded RFs, the centripetal Surround-Only AM

449. response along the preferred orientation axis (CP-ISO, left and right red waveforms) is the strongest

450. among the responses evoked by the Surround stimulation alone : compare it with the CP-CROSS

451. conditions along the RF width axis (top and bottom, gold waveforms), for which responses are much

452. weaker.

453.

454. Fig 3. Spatio-temporal and axial selectivity of "Surround-Only" responses to Centripetal AM flow

455. [A] Single Cell example : Central inset cartoon: ON and OFF discharge fields, respectively in red and blue. White

456. contour delineates the subthreshold receptive field (SRF). For comparison, respective positions of the Gabor

457. inducer stimuli flashed in the surround are overlaid on the RF map. Two-stroke "Surround-Only" AM responses :

458. Comparison of "collinear" (red) and "parallel" (gold) responses evoked respectively along the orientation

459. preference and across the width axis of the RF, with the Center-only response (black waveform). Bottom left

460. corner, Vm subthreshold responses for sparse noise (ON (dark red) and OFF (dark blue) waveforms).

461. [B] Dependency of Surround-Only responses on the orientation of the inducer : Right, top and bottom panels

462. illustrate the Gabor inducer features and the color codes for the motion axis explored respectively in the ISO-

463. and CROSS-configurations. The color code conventions are : ISO-RF: red for "collinear" along the orientation

464. preference axis; gold for "parallel" across the RF width axis; CROSS-RF: cyan for "parallel" across the orientation

465. preference axis; pink for "collinear" along the RF width axis. See Text for details. 
466.

467. A complete study of the dependency of the Surround-Only AM responses on the GP inducer

468. orientation relative to the RF preferred orientation was replicated in 13 cells. The average responses

469. of this population have been computed by realigning arbitrarily the orientation and direction

470. preference of each recorded cell respectively with the horizontal and right pole axes. The mean

471. population tuning responses are illustrated in Fig. 3B, where the different AM flow conditions are

472. schematized by color-coded arrows in the two right inserts. The top insert regroups the specific

473. centripetal AM flows where the inducer is either "collinear" (red) or "parallel" (gold)) with the RF

474. preferred orientation (ISO-RF condition). The bottom insert details the stimulation cases where the

475. inducer is orthogonal with the RF preferred orientation (CROSS-RF: cyan along the main axis, pink

476. along the RF width axis).

477. This study reveals two effects of the Surround contribution. The first one is a non-specific

478. facilitation component found for all AM configurations, regardless of the orientation of the inducers

479. relative to that of the RF. It corresponds to the fact that all centripetal Surround-Only flows, which

480. terminate in the "Near" periphery of the RF core, recruit simultaneously synaptic input originating

481. from the feedforward SRF as well as proximal horizontal input. The second one is orientation selective

482. and reflects the dominance of responses evoked by centripetal flows of Gabor elements "co-aligned"

483. with the RF preferred orientation (Fig. 3B) : the evoked depolarizing Surround-Only subthreshold

484. response elicited by CP-ISO collinear flow along the main RF axis (red trace, horizontal axis in all

485. figures) is, on average, 2.6 times larger than that evoked by CP-ISO- (pink) or CP-CROSS- (gold) flows

486. along the RF width axis and by CP-CROSS- flows (cyan) across the RF main axis.

487. The classically reported "edge-detector" property of V1 neurons, as initially described by Hubel

488. and Wiesel (46), highlights the fact that the strongest discharge is evoked by "parallel" elements swept

489. towards the RF center orthogonally across its width axis (CROSS_width-axis configuration, gold trace,

490. right top inset in Fig. 3B) at low speed (1-10\%). In contrast, the detailed comparison between the CP-

491. ISO main-axis (red traces) and the CP-CROSS width-axis (gold traces) configurations implemented here 
492. illustrates a functional switch of preferred integration axis between low-speed and high-speed

493. recruitment of surround sources. Indeed, at high AM speed (100-500\% $/ \mathrm{s})$, the synaptic integration of

494. "Near" periphery was found to depend on the motion axis, revealing a spatial selectivity and

495. anisotropy not present in the MDF mapped with light/dark impulses. Furthermore, the centripetal

496. flow of co-aligned GPs along the RF preferred orientation axis (CP-ISO) revealed to be the most

497. efficient in inducing a tonic subthreshold enhancement of sensory responsiveness irrespectively

498. of the RF center stimulation (or its omission). Finally, the comparison of the CP-ISO and CP-CROSS

499. mean subthreshold response profiles shows a clear orientation and axial bias of the "Surround-only"

500. responses for Gabors co-aligned along the orientation preference axis (red waveforms). In contrast, an

501. absence of local feature selectivity was found for AM along the RF width axis (pink and gold traces),

502. with response levels similar to that evoked by CROSS-oriented elements along the RF main axis (cyan

503. traces). In addition, the recruitment of the "Near" periphery rose significantly the level of excitability

504. of the recorded cells (one sided $Z$-test above resting state, $p<0.05$ ). A mean integral depolarization

505. was apparent in 76\% of cells for the CP-ISO "collinear" (red) condition, and in 55\% in the "parallel"

506. (gold) condition at high AM speed. Considering both main and width axes together, independently of

507. the efficiency with which the "Near" surround was recruited, even spiking facilitation was observed in

508. $59 \%$ of the cases while leaving the MDF center unstimulated.

509. Altogether, the comparative analysis between each configuration of Surround-Only stimulation

510. validates the prediction of the "Dynamic Association Field" hypothesis, a concept defined in our

511. previous study (29): the collinear configuration promotes input collection along the orientation

512. preference axis, resulting in the emergence of centripetal axial direction selectivity for high-speed co-

513. aligned stimuli.

514.

\section{2. Specificity of the interaction between "local" (inducer) and "global" (AM motion) features}




\section{2.1. Inducer co-alignment with the RF orientation promotes centripetal synaptic integration along the global motion axis}

517. The following contextual analysis focuses now on the modulatory effect that a two-stroke

518. activation of the silent Surround (along each of the cardinal axes) can produce on the response to a

519. subsequent test Gabor stimulus (the third stroke), flashed at the preferred orientation within the MDF

520. (right column in Fig. 1B). In addition to stimulations restricted to the Surround, the contextual impact

521. of the Surround was measured (in an interleaved way) by comparing the response to the full apparent

522. motion sequence terminating in, or departing from the RF (3-stroke AM, centripetal (CP) "Surround-

523. then-Center" and centrifugal (CF) "Center-Then-Surround" conditions) to the response to the test

524. stimulus in the RF ("Center-Only" condition).

525. The complete set of configurations including Centripetal (CP) and Centrifugal (CF) flows of Gabor

526. inducers either co-aligned with the motion axis along the RF preferred orientation ("collinear"), or

527. orthogonal to the RF width axis ("parallel"), was tested in 23 cells. As expected from the feature

528. characteristic of Surround-Only responses, both "collinear" and "parallel" centripetal AM flows

529. invading the RF elicited a facilitating effect on the Center test response. Considering centripetal flows,

530. a significant depolarizing facilitation by the surround stimulation could be observed respectively in

531. 95\% of occurrences for "collinear" and 69\% for "parallel" conditions. A similar observation was

532. observed at the spiking level, with respectively $84 \%$ of occurrences for "collinear" and $66 \%$ for

533. "parallel" conditions (one sided Z-test above resting state, $p<0.05$ ).

534. Fig. 4 illustrates the case of a highly selective dependency of contextual control of the cortical

535. transfer function on the flow direction, emphasizing the functional facilitatory impact of centripetal

536. collinear (CP-ISO) flows (red profile, upper panel) proceeding from Surround to Center along the

537. orientation preference axis of the RF: in addition to the change in response integral, a significant

538. latency shortening is observed both at the subthreshold (upper waveforms) and spiking (lower filled

539. PSTHs) levels (compare with black profiles of the Center-Only response). In contrast, a late change in

540. the PSTW waveform integral, but not of its latency, is observed when the AM flow is centrifugal (CF) 
bioRxiv preprint doi: https://doi.org/10.1101/2021.09.10.459746; this version posted September 11, 2021. The copyright holder for this preprint (which was not certified by peer review) is the author/funder. All rights reserved. No reuse allowed without permission.

Horizontal propagation of "network belief" in V1

20 August 2021

541. and proceeds from Center to Surround (green and brown, bottom panel of Fig. 4). These differential

542. kinetic changes fit with those expected from a simple summation model, since CP- and CF-AM

543. sequences respectively impact the early and late phase of the response when stimulation of the

544. Surround precedes the Center or when the temporal order of stimulation is reversed.

545.

546. Fig. 4 : Example of contextual modulation of the Center-response by Centripetal AM ("cardinal” protocol).

547. Example of Center-Surround AM responses, compared to the Center-Only condition. The comparison, in the

548. same cell, between centripetal (CP: top panel) and centrifugal (CF: bottom panel) motion flows illustrates the

549. importance of the Surround-then-Center timing in the associative effect. PSTWs (top) and PSTHs (bottom) for the

550. four AM axes (red (CP) and green (CF), "collinear" along the RF orientation preference axis; gold (CP) and brown

551. (CF), "parallel" across the RF width axis. The contextual response is overlaid on the Center-Only stimulation

552. (black). Note that the contextual latency shortening and the spike discharge rate increase of the Center response

553. are observed only in the collinear Centripetal condition (top, red).

554.

555. Considering both centripetal and centrifugal flows, among the pool of 23 recorded cells, a strong

556. bias for "collinearity" was observed in $91 \%$ of the cells at the subthreshold level and $95 \%$ at the

557. spiking level (the bias was averaged across the two RF sides for the same axis). In terms of magnitude,

558. the "collinear" AM flow produced the largest modulatory effects and evoked, on average, a

559. depolarizing response 2.6 times larger and a spiking response 2.0 times stronger, than the "parallel"

560. sequence condition. At the spiking level, effects varied from cells to cells, but, in most cases, they were

561. characterized by a shortening of spiking latency along the "collinear" axis for centripetal flows.

562. Changes in firing rate integral or response integral mainly affected the "collinear" axis response, but,

563. as expected from the non-specific component of Surround-only responses, facilitation was also

564. observed for the "parallel" stimulations : note the weak CROSS_width-axis "parallel" facilitation seen

565. for centripetal (top panel, gold waveforms) and centrifugal traces (bottom panel, brown waveforms of

566. Fig. 4). 


\section{2.2. Differential impact of "Near" and "Far" Surround recruitment}

568. In order to better delineate the contribution of the "Far" periphery, we designed a more

569. conservative stimulation protocol (the "radial" protocol, Fig. 1C) where the Surround stimulation was

570. allowed only outside the impulse SRF (and not only the MDF, as done previously in the "cardinal"

571. protocol). The spacing between GP inducers was increased from $120 \%$ of the MDF to $100 \%$ of the SRF

572. and the absence of overlap between the nearest Surround stimulus and the SRF was checked for each

573. of the recorded cells. Stimulation was no longer restricted to the main and cardinal axes but extended

574. to angular SECTORS surrounding the original cardinal axes by two neighboring $\left( \pm 30^{\circ}\right)$ stimulation axes,

575. mimicking the spatial sensitivity of the synaptic association field (29). We also dropped, from the

576. stimulation set, the AM configurations used in the cardinal protocol for which non-specific component

577. responses were evoked (when co-varying the local inducer orientation and the motion axis). This

578. enabled us to focus on our main conditions of interest with GP elements ISO-oriented with the motion

579. axis always presented along the RF preferred orientation axis (CP-ISO and CF-ISO) while elements

580. CROSS oriented to the motion axis only referred to stimulations along the width axis of each recorded

581. cell (CP-CROSS).

582. Note here the terminology change in ISO and CROSS definitions, imposed by the radial symmetry

583. introduced in this latter protocol. When applied to the formal (cardinal) protocol, the ISO-axis

584. conditions correspond to cases where the orientation of the flashed Gabor patches is either "co-

585. aligned" with the RF preferred orientation axis (1st row in Fig. 1B; CP- and CF-ISO_main-axis, red and

586. green arrows), or cross-oriented with it, but, in that later case, co-aligned the width axis (CP-

587. ISO_width-axis, pink). Accordingly, the CROSS-axis conditions (CP-CROSS_main-axis and CP-

588. CROSS_width-axis) are respectively schematized by cyan and gold arrows for CP-AM and brown arrows

589. for CF-AM flows in Fig. 1B). In addition, in the "radial" protocol, each of the 37 cells recorded was

590. stimulated with an extended benchmark of contextual 5- to 6-stroke AM flows, richer than in the

591. cardinal protocol. The parametric systematization of the various flows of ISO-axis or CROSS-axis GP

592. sequences is detailed in each row of Fig. 1C. 
593. Fig. 5 illustrates important spatial differences in the Surround periphery recruitment between the

594. two protocols ("cardinal" in A; "radial" in B), by comparing the respective time-course profiles of the

595. intracellular responses, evoked by the sole stimulation of the surround (Surround-Only, dotted traces)

596. and by the complete AM sequence terminating in the RF center (Surround-then-Center, continuous

597. traces). The top row in Fig. 5 illustrates the RF maps of the spiking discharge fields (ON (red) and OFF

598. (blue)) and of the subthreshold SRF (topological union of ON and OFF depolarizing fields) delimited by

599. a white contour. In order to focus the comparison with the stimulation axes used in the "cardinal"

600. protocol, we illustrate, for the "radial" protocol cell (B, right panel), only the CP-ISO and CP-CROSS

601. Sector AM configurations (first and third row, in the left column of Fig. 1C, red and gold schemes)

602. along respectively the RF main and width axes. A straightforward observation directly emerges from

603. the comparison of the visual field paving with Surround GPs applied in each protocol : for the same

604. spatial viewing field size centered on each $\operatorname{RF}\left(20^{\circ} \times 20^{\circ}\right)$, the two Surround sources of stimulation are

605. visible for the "cardinal" protocol, while only one - the most proximal (to the RF center) - appears for

606. the "radial" protocol. The closest GP location in the periphery (D1 position in Fig. 1A) partially

607. encroaches on the border of the SRF in the "cardinal" protocol, while this is not the case (by design)

608. for any surround sources in the "radial" protocol.

609. This difference in the spatial overlap factor accounts for the differences in intracellular response

610. magnitudes observed in each protocol. In the "cardinal" case (Fig. 5A), the "Near" Surround-Only

611. stimulation evokes sizeable depolarizing responses, both for CP-ISO and CP-CROSS stimulations

612. (dotted red and gold waveforms). Both types of CP Surround-then-Center AM flows strongly amplify

613. the evoked PSP amplitude although the peak amplitude gain is the largest in the CP-ISO condition. In

614. contrast, the typical behaviour observed in the "radial" protocol (Fig. 5B) shows a different profile.

615. For a comparable magnitude of the Center-only response (8.0 mV ("radial") vs. $7.4 \mathrm{mv}$ ("cardinal")),

616. the purely modulatory influence of the CP-ISO AM flow restricted to the "Far" Surround was generally

617. much weaker (2-3 times) than that seen for the "cardinal" protocol (here, $3.9 \mathrm{mV}$ ). In contrast to the

618. CP-ISO condition, the CP-CROSS Surround-Only stimulation is almost inefficient in inducing a 
bioRxiv preprint doi: https://doi.org/10.1101/2021.09.10.459746; this version posted September 11, 2021. The copyright holder for this preprint (which was not certified by peer review) is the author/funder. All rights reserved. No reuse allowed without permission.

Horizontal propagation of "network belief" in V1

20 August 2021

619. contextual response (compare the dotted red and gold traces in the bottom right panel in Fig. 5B).

620. As a consequence, in the "radial" protocol, the complete CP-ISO AM sequence (terminating in the RF

621. center) is the only condition inducing a phase advance and a significant amplification of the early

622. Center-only response.

623.

624. Fig.5 : Differential recruitment of "Near" vs "Far" Surround between the "cardinal" vs. "radial" protocols

625. [A]: "cardinal" protocol, and [B]: "radial" protocol. Top row, receptive field maps. Same convention as in Fig.

626. 3A. Only the Surround GPs on the RF main (horizontal) and width (vertical) axis (common to both protocols) are

627. represented within the same viewing field $\left(20^{\circ} \times 20^{\circ}\right)$. Note the encroachment of the most proximal GPs in the

628. "cardinal" protocol over the SRF (white contour), whereas no spatial overlap is seen in the "radial" protocol.

629. Bottom rows, the CP-ISO (red) and CP-CROSS (gold waveforms) are overlaid for the "Surround-Only" (dotted)

630. and the "Surround-then-Center" (continuous trace) conditions and compared to the "Center-Only" test condition

631. (black). Insets show respectively an expanded version of the PSPs onset on a 30ms time window. Arrows indicate

632. the change in PSP slope due to the feedforward synaptic drive triggered by the Center test stimulus. See Text for

633. details.

634.

635. A mechanistic difference between the two protocols is further attested by the comparison of the

636. rising slopes of the time-profiles of the AM responses with that of the feedforward response (Center-

637. Only). In the "cardinal" example cell (bottom left panel in Fig. 5A), both the CP-ISO Surround-Only and

638. the CP-ISO Surround-then-Center evoked PSPs have the same initial rising slope and latency (in

639. absolute value terms, relative to D1 stimulation) as the Center-Only response (relative to D0

640. stimulation), thus suggestive of a synaptic feedforward input signature. Note also that both the CP-ISO

641. and CP-CROSS synaptic responses start to rise simultaneously in the "cardinal" case, which indicates

642. that the "Near" stimulated periphery overlaps with the feedforward synaptic drive. In contrast, in the

643. case of the "radial" example (bottom right inset in Fig. 5B), the CP-CROSS profile is undistinguishable

644. from that of the Center-Only (gold and black) waveform and the initial rising slope of the CP-ISO 
645. response (red) occurs much earlier and is initially weaker than that of the Center-Only response. These

646. latter features are suggestive of a slower spatio-temporal diffusion process compatible with horizontal

647. activity propagation. This qualitative comparison clearly emphasizes that preventing the closest

648. surround-location from encroaching on the SRF border leads to the disappearance of the non-specific

649. tonic component of the boosting of cortical responsiveness seen in the "cardinal" protocol.

650. Remarkably enough, on the whole population, the "radial" protocol led to a drastic decrease of i)

651. the proportion of cells showing "Surround-Only" responses (32\% (“radial”) vs. $100 \%$ (“cardinal”), ii)

652. the magnitude of Surround responses, that never reached the peak amplitude of the Center-Only test

653. response, and iii) the evoked spiking level in response to the complete "Surround-then-Center" CP-

654. AM. Although the collinear configuration ("CP-ISO") remained the most effective in the "radial"

655. protocol, the observation of a tonic non-specific component (seen for all conditions of the "cardinal"

656. protocol) was not reproduced for Surround-Only responses and consequently, their facilitation role

657. was weaker at both Vm and Spiking levels. A likely explanation is that, in contrast to the "cardinal"

658. protocol, the "radial" stimulation of the Surround no longer recruits as efficiently the "Near"

659. periphery, which seems to integrate both lateral and feedforward input. Importantly, the relative peak

660. amplitude and timing of those responses regarding control contextual stimulations, in sequences

661. restricted to the Surround or invading the RF Center were much more specific. Consequently, the

662. "radial" protocol appears best tailored to detect the selective contribution of purely lateral

663. recruitment of the "Far" periphery.

\section{2.3. Anticipatory "filling-in" responses ("radial" protocol)}

665. To highlight the emergence of a lateral anticipatory wave due the sole recruitment of the distal

666. periphery (Surround-Only wave) and quantify its functional role in the contextual gain control of the

667. response evoked by the feedforward drive, we determined - in the "radial" protocol - the individual

668. significance of each cell response to the Surround-only condition, using a point-by-point

669. randomization test $\left(10^{4}\right.$ repetitions, $\left.p<0.01\right)$. The largest proportion of cells showing significant

670. "Surround-Only" responses was found for the CP-ISO condition ( 12 cells ( $32 \%$ of the population)). A 
bioRxiv preprint doi: https://doi.org/10.1101/2021.09.10.459746; this version posted September 11, 2021. The copyright holder for this preprint (which was not certified by peer review) is the author/funder. All rights reserved. No reuse allowed without permission.

Horizontal propagation of "network belief" in V1

20 August 2021

671. single cell example is illustrated in the left column of Fig. 6. A complete CP-ISO sequence invading

672. the RF Center reveals a clear boosting effect (compare continuous red (AM) and black (Center-Only)

673. traces, top and bottom panels). In spite of the fact that the closest surround location was sparing the

674. SN-mapped SRF, a sizeable significant change in membrane potential was still observed in the AM

675. sequence restricted to the surround, from D5 to D1 locations, in the absence of D0 stimulation

676. (bottom left graph in Fig. 6, dashed red trace). For this cell, the combined dynamic recruitment of

677. the GP sources along the AM motion axis shows a progressive build-up signal which anticipates, by ten

678. milliseconds, the onset of the feedforward signal evoked by the Center-stimulus. In contrast, the linear

679. predictor based on individual static responses to GPs in isolation does not reach significance at the

680. time where center-only response departs from resting activity (bottom left graph in Fig. 6; dashed

681. green trace). Thus, in this particular single cell example, the AM sequence recruits a non-linear

682. amplification process of subliminal sources, attesting for the role of spatio-temporal synergy in

683. maximizing the functional impact of horizontal propagation.

684.

685. Fig.6 : "Filling-in" responses evoked by "Surround-Only" AM ("radial” protocol).

686. The analysis here is restricted to cells where "Surround-Only" stimulation evoked a significant response $(n=12$,

687. see Text for criteria). Color code: black for "Center-only"; red for "Surround-then-Center", dashed red for

688. "Surround-Only". The middle horizontal insets respectively show the chronograms of the stroke-by-stroke

689. stimulation sequences for "Center-Only" (1 stroke, filled black box, upper line), "Surround-Then-Center" (6

690. strokes, filled red, middle line) and "Surround-Only" (5 strokes, filled pale red, bottom line) protocols. Empty

691. boxes indicate omitted stimuli. The time onset of each GP stroke is labeled by a thin dotted vertical line. In this

692. figure and the following ones, the blue dot and vertical dotted blue bar on the $y$-axis indicate the "threshold"

693. amplitude change of the Center-Only control curve from rest, above which statistical significance of the response

694. is reached $(p<0.01)$ for each individual cells. Their abscissa serves as a reference for the temporal realignment of

695. the different contextual responses. Left, top panel : Single cell example. The complete CP-ISO AM sequence (D5

696. to D0) evokes a significant facilitation of the "Center-Only" Vm response (compare red and black traces). Bottom

697. Left : when omitting the D0 stroke, the recruitment by the CP-ISO AM flow, although limited to the silent 
bioRxiv preprint doi: https://doi.org/10.1101/2021.09.10.459746; this version posted September 11, 2021. The copyright holder for this preprint (which was not certified by peer review) is the author/funder. All rights reserved. No reuse allowed without permission.

Horizontal propagation of "network belief" in V1

20 August 2021

698. surround (D5 to D1), still induces a depolarizing activation ("Surround-Only": dashed red). The temporal profile

699. of the lateral wave of activity matches the "predicted" invasion of the RF Center, had it been stimulated. This

700. effect, in this cell, results from a synergistic interaction, since it cannot be explained by the sum of the static

701. responses evoked by each distal GP stroke in isolation (dashed green, undistinguishable from rest activity).

702. Right, top panel : Average response profiles for "Surround-then-Center", "Center-Only" and "Surround-Only"

703. conditions $(n=12)$. Right, bottom panel : the CP-ISO Surround-Only response (mean: dotted red \pm SEM: shaded

704. area) is compared (with a different ordinate scale than in the top right panel) with the "expectation" (dashed

705. black trace) obtained by subtracting the Center-Only (black trace, Top right) from the complete CP-ISO AM

706. response (red trace, Top right). See Text for interpretation.

707.

708. In the subpopulation of cells showing significant CP-ISO Surround-Only responses (point by point

709. permutation test, $10^{4}$ repetitions, $\left.p<0.01\right)$, statistical analysis revealed that $6 / 12$ cells $(50 \%$ of

710. significant cases; $16 \%$ of the entire population) showed significant anticipatory responses larger than

711. their static linear predictor in the CP-ISO condition. For Surround-Only significant cases, the CP-ISO

712. Surround-Only AM sequence triggered a synaptic wave riding clearly above the noise level, peaking at

713. an average value corresponding to a third of the Center-Only test response peak amplitude for an

714. absolute value ranging between 1 to $4 \mathrm{mV}$ (average dotted red trace in top right panel of Fig. 6).

715. Remarkably for the optimized AM speed, the Surround-Only average response profile rose peaked at

716. the time of expectation of the Center-Only response. This strongly suggests a causal relationship

717. between purely contextual information and its integration in the gain control of complete sequences

718. terminating in the RF center.

719.

\section{3. Dependency of the contextual control of the cortical transfer function on the spatio-temporal coherence of the AM flow}

721. In order to systematically identify and quantify the key spatial and temporal factors of the CP-ISO

722. AM sequence which conditions the contextual control of the cortical gain, we compared for each of

723. the 37 cells, the CP-ISO configuration with AM sequences in which the spatio-temporal structure of the 
bioRxiv preprint doi: https://doi.org/10.1101/2021.09.10.459746; this version posted September 11, 2021. The copyright holder for this preprint (which was not certified by peer review) is the author/funder. All rights reserved. No reuse allowed without permission.

Horizontal propagation of "network belief" in V1

20 August 2021

724. GP presentation was altered (Fig. 1C). The statistical significance of the measures extracted from the

725. mean $\mathrm{Vm}$ responses of individual cells (top panel in Fig. 7) was assessed by comparing each AM

726. condition to the same reference (Center-Only) independently for the two criteria of onset latency and

727. response strength changes, and in addition, in a less stringent way, for one or the other criteria

728. (randomization test, $10^{4}$ repetitions, $\mathrm{p}<0.05$ ). Both congruent and complementary trends were found

729. across the three statistical criteria we used.

730.

731. Fig. 7. Statistical significance of contextual response changes

732. Top panel, ranked distributions $(n=37)$ of changes in Latency (ms, left column) and in response integral value

733. (normalized ratio, right column) of subthreshold responses (Vm), for each SECTOR AM condition of interest (first

734. three rows). The fourth row corresponds to the FULL-RND condition (see Text for justification). Color code, same

735. as in Fig. 1C, schematized by appropriate icons in the right colum. Abscissa, cell rank (1 to 37 ), done

736. independently for each AM condition. Filled bars represent the amplitude change of "significant" individual cases

737. (one-sided permutation test, $10^{4}$ repetitions, $p<0.05$ ). Ordinates: left column, latency change (" $\Delta$ Latency")

738. measured at half-height of the Center-Only peak response; right column, change in the integral value of

739. "significant" depolarization $\left( \pm 3 \sigma\right.$ from mean $V_{\text {rest }}$ ), measured from the onset of the test GP flashed in the RF

740. Center to the point of return to baseline of the Center-Only response. The integral value change (" $\Delta$ Response“)

741. is expressed as a normalized ratio relative to the Center-Only reference response.

742. Bottom panel, each of the three histograms represents the proportions of significant cases for each AM flow

743. condition (same color code as in Fig. 1C). Left, " $\Delta$ latency" criteria. Right: " $\Delta$ Response" criteria. Center inset

744. (ВОTH): all cases showing either a significant latency advance, or response integral increase, or both.

745.

746. For latency changes only (ranked distributions, left column in the top panel of Fig. 7), the

747. proportion of significant cases is three times larger for the CP-ISO condition (30\%, red column in the

748. left histogram, bottom panel) than for the other conditions. For response integral changes only

749. (ranked distributions, right column in the top panel of Fig. 7) the effect is less selective across

750. conditions, but remains specific of the collinear configuration, since most significant cases are found in 
bioRxiv preprint doi: https://doi.org/10.1101/2021.09.10.459746; this version posted September 11, 2021. The copyright holder for this preprint (which was not certified by peer review) is the author/funder. All rights reserved. No reuse allowed without permission.

Horizontal propagation of "network belief" in V1

751. both CP-ISO and CF-ISO conditions (respectively red and green bars in the right histogram, bottom

752. panel). When pooling both criteria in a non-exclusive way (logical "OR", bottom center inset), the

753. proportion of significant cases peaks for the CP-ISO condition (red histogram bar, 41\%), while

754. remaining two times larger than for the CF-ISO condition (19\%, green bar) and three times larger than

755. the other conditions (11\%, gold bar for CP-CROSS; $14 \%$, blue bar for RND-ISO). These different analyses

756. show that the CP-ISO AM flow is by far the most effective configuration to promote the visibility of

757. Surround-Center interactions.

758. For the CF-ISO condition, no latency advance effect was induced at the PSP level on the

759. population average $(n=37)$, since the rising phase of the $C F$-induced $V m$ response, as expected, was

760. undistinguishable from the Center-Only response. Only $8 \%$ of the cells showed a significant latency

761. advance of a modest magnitude in the CF-ISO condition compared to the $30 \%$ found in the CP-ISO

762. condition (green bar vs red in the left histogram, bottom panel of Fig. 7). Conversely, contextual

763. facilitation of the Center response integral was present in a slightly larger proportion of cells (14\%,

764. green bar in the bottom panel right histogram of Fig. 7). Those observations concord with those of

765. the "cardinal" protocol, since the rising phase of the CF-induced response, as expected, was

766. undistinguishable from the Center-Only response. On the other hand, the increase in response integral

767. value concords with a lasting depolarization following the center-only test peak response, as more and

768. more Gabor patches flashed at increasing eccentricity from the RF center evoke a series of lagged

769. desynchronized lateral inputs of exponentially decreasing amplitude. This first control demonstrates

770. that the spatial co-alignment of GPs is not enough in itself to induce the observed boosting effect.

771. Some form of spatio-temporal ordering is required such that an anticipatory flow from the periphery is

772. generated, which, in fine, will boost sensory responsiveness at the time when the test stimulus hits the

773. RF Center.

774. Since, whatever the speed, moving cross-oriented stimuli across the orientation preference axis of

775. the recorded RF were in most cases ineffective in firing the cell, we studied the impact of CP-CROSS

776. AM sequences along an angular sector restricted around the width axis (Fig. 1C: left column "SECTOR"; 
bioRxiv preprint doi: https://doi.org/10.1101/2021.09.10.459746; this version posted September 11, 2021. The copyright holder for this preprint (which was not certified by peer review) is the author/funder. All rights reserved. No reuse allowed without permission.

777. gold condition). This however implies in the SECTOR configuration that the analysis relies on the

778. comparison of response flows (CP-ISO vs. CP-CROSS) sweeping across different surround subregions,

779. while maintaining the orientation of all GPs the same. Nevertheless, the absence of an orientation-

780. selective component for centripetal flows of CROSS-oriented GPs along the main axis of the RF (CP-

781. CROSS_main-axis, cyan traces in Fig. 3) justifies this simplification choice. In spite of these subtle

782. protocol differences, only $11 \%$ of the cells showed a significant positive latency advance to the CP-

783. CROSS stimulation, while none displayed any significant change in the response integral, when

784. compared to the Center-Only test response (bottom panel, respectively left and right gold histogram

785. bars in Fig. 7). Overall, the weak changes in latency advance or amplification of the Center-Only

786. response found in individual cells during CP-CROSS AM flows was washed out completely when

787. averaging across cells (RIGHT panel in Fig. 8, compare yellow and black mean waveforms). A similar

788. conclusion is reached whether the surround was partially (SECTOR) or uniformly (FULL) recruited: in

789. both conditions (SECTOR and FULL), the contextual mean CP-CROSS responses were almost

790. undistinguishable from the Center-Only test response (data not shown).

791.

792. Fig. 8 : Contextual Gain Control induced by centripetal-ISO AM flow ("radial" protocol)

793. Top row, subthreshold response (Vm); Bottom row, firing rate response (spikes/s). The first three columns, from

794. the left illustrate the boosting effect produced by a CP-ISO AM sequence (red curve, "Surround-then-Center")

795. when compared to the response to the test GP inducer flashed in the RF Center (black curve, "Center-Only"). The

796. local orientation of the GP inducers in the Surround and the global AM axis are co-aligned with the RF

797. orientation preference. The last (right) column focuses on contextual dependency of the effect.

798. From left to right, first column : Single cell example, where the vertical dotted gray lines indicate the respective

799. timing of each peripheral stoke stimulation at successive eccentricities (from D5 to D1), whereas the continuous

800. vertical gray line indicates the onset of the "Center-Only" test stimulation (D0). The blue dot and corresponding

801. dotted line give the latency of the first point in time where the "Center-Only" response departs significantly from

802. the resting state $(p<0.01)$. Second column from the left, population averages for the CP-ISO (red) and

803. the Center-Only (black) conditions. Averaging is done in two steps, first across trials for each cell for the same 
bioRxiv preprint doi: https://doi.org/10.1101/2021.09.10.459746; this version posted September 11, 2021. The copyright holder for this preprint (which was not certified by peer review) is the author/funder. All rights reserved. No reuse allowed without permission.

Horizontal propagation of "network belief" in V1

20 August 2021

804. context condition, then across cells after realigning individual mean waveforms around a common onset latency

805. of their "Centre-Only" response (" 0 " mark of the "Relative Time" abscissa, blue points in left panels). Response

806. amplitudes are normalized relatively to the peak of the "Center-Only" response. The third column illustrates,

807. for "significant cells" only (see Fig. 7), the difference in rising phases and onset latencies between the CP-ISO

808. (red) and Center-Only responses on an expanded time basis. The mean \pm SEM value envelopes are respectively

809. indicated by a continuous curve and a shaded area. The third column shows the same trend for the whole

810. population, all cells included, irrespectively of the significance criteria. Right column, the significance analysis is

811. extended to cells which showed significant Surround-only responses (see Text for criteria: subthreshold: $n=20$;

812. spiking: $n=10$ ). The contextual averages (color code same as in Fig. 1C) show that CP-ISO (red) is the only

813. condition where a change in onset latency is observed, both at the Vm (top) and spiking levels.

814.

815. In a third control condition, the same retinal SECTOR space as in our CP-ISO condition of interest

816. was stimulated with the same GPs (i.e., with the same number, features, and stimulus energy

817. distribution) but the coherence of the flows was pseudo-randomized both in space and time. Such

818. RND-ISO condition induced a slight facilitation effect on average, although significantly smaller than

819. the one seen in the CP-ISO condition. We explored the possibility that this partial effect could be of

820. residual nature and come from an insufficient level of randomization achievable in the SECTOR

821. stimulation. Indeed, the number of visited nodes (i.e. GPs) is rather low ( $n=26)$, with the additional

822. constraint that the last Gabor patch in the sequence had to be the one flashed in the Center. Note

823. that, in contrast to coherent-AM sequences, GPs flashed during each step of the RND-ISO AM

824. sequence could belong to any ring of eccentricity, thereby increasing at each step of the AM sequence

825. the probability to find D1- (or D2-) to center stimulations (see Methods). Close examination of

826. individual cell recordings showed that the slight facilitation most likely reflected the fact that in some

827. RND-ISO trials, the penultimate GPs were presented occasionally by chance at the D1, or even D2

828. eccentricity (Fig. 1A), just one stroke ahead of the RF center stimulation. We concluded that the Sector

829. RND-ISO sequence often recruited facilitating two-stroke AM Surround-Center pairs whose effect has

830. already been reported (see fig. 7 in (29)). To dilute the remnant impact of proximal-to-center 
bioRxiv preprint doi: https://doi.org/10.1101/2021.09.10.459746; this version posted September 11, 2021. The copyright holder for this preprint (which was not certified by peer review) is the author/funder. All rights reserved. No reuse allowed without permission.

Horizontal propagation of "network belief" in V1

20 August 2021

831. interactions seen in the RND SECTOR condition, we used, as a more randomized control, the FULL

832. stimulation configuration in which the probability to find such co-aligned D1 (or D2) GP followed by

833. the RF center stimulation during the entire stimulation protocol was lowered by increasing the

834. number of possible peripheral node locations (from $n=26$ to $n=52$ ), thereby increasing the level of

835. randomization of the AM sequence. In the RND FULL mode, the stimulation was not restricted to

836. retinal sectors on both sides of the RF but was extended isotropically to its entire Surround (bottom

837. right blue icon in Fig. 1C). In a limited fraction of the cells, response to the FULL RND stimulation still

838. displayed a slight facilitatory effect ( $11 \%$ for latency change and $3 \%$ for response gain) compared to

839. the Center-Only test response (blue columns, in respectively the left and right histogram bars in the

840. bottom panel of Fig. 7), and this effect disappears when averaging across cells (compare average blue

841. and black waveforms in the right panel in Fig. 8). We conclude that the contextual boosting of the

842. cortical transfer function was largely washed out by the reduced probability of observing collinear

843. proximal activation in the FULL RND sequences, resulting from the more extensive randomization of

844. the flow's spatio-temporal coherence than in the SECTOR RND condition.

845. Let us focus more in depth on the CP-ISO condition. The left column in Fig. 8 illustrates the typical

846. contextual behaviour observed for Vm and spiking responses at the single cell level for the CP-ISO

847. configuration in the "radial" protocol, when compared to the Center-Only condition: the AM CP-ISO

848. sequence produces an anticipatory depolarizing wave that shortens the onset latencies of both the

849. rising phases of the PSP (upper row) and the spiking response (bottom row), by respectively 14.7 and

850. $16.3 \mathrm{~ms}$. For that particular example, both a progressive build-up of a subthreshold depolarization and

851. an anticipatory firing response are observed at early latencies, but this ramp-up feature was not

852. systematically observed in all cells. At the population level, we found that centripetal flows composed

853. of Gabor elements co-aligned with the global motion path along the RF main axis (CP-ISO) resulted in

854. an overall boosting of the neural response compared to that evoked by the test Center stimulus alone.

855. This finding is illustrated in the second column (from the left) of Fig. 8. Note here that the PSTW and

856. PSTHs realignments (see Fig. 6 legend) are done separately for the averaging since a few millisecond 
857. integration lag is found between the absolute measurements of significant onset times for $V m$ and

858. spikes for the Center-Only responses (see the causal shift in the vertical dotted blue lines in the single

859. cell example). On average ( $n=37)$, the CP-ISO stimulation, in the SECTOR condition, resulted in an

860. overall latency shortening and a slight amplification of the depolarizing subthreshold response

861. envelope ( $\mathrm{Vm}$, upper row). It also led to a reduction in latency of the spiking discharge (third column,

862. bottom panel) in cells where spiking activity evoked in the Center-Only condition was significant

863. (permutation test, $10^{4}$ repetitions, $p<05 ; n=22$ ).

864. In order to better quantify the effect, we restricted, in a second step, the averages to the group of

865. "significant" cells for which significant changes in latency ( $\Delta$ latency) and/or strength ( $\Delta$ response

866. integral) of the subthreshold component of the Vm response were induced by the CP-ISO AM flow (see

867. Methods and Fig.7). Results show that latency change and response integral modulation by the

868. centripetal AM flow significantly facilitated responses of $41 \%$ of the cells (15/37) of the CP-ISO

869. condition. The third column in Fig. 8 focuses on the rising phases of the CP-ISO and Control

870. waveforms, and illustrates, with an expanded time basis, the significant changes in Vm and spiking

871. latencies. Note that the quenching around the " 0 " time abscissa, seen for the Center-Only responses,

872. results from the realignment process applied before averaging (see Methods).

873. To further demonstrate the contextual boosting specificity concomitantly at the synaptic and the

874. firing levels, we tried to maximize the population size. For this last analysis, we added - to the pool of

875. CP-ISO "significant" cells (based on Fig. 7 criteria) - the contingent of cells for which significant

876. responses could be elicited by an AM flow stimulation limited to the Surround (permutation test, $10^{4}$

877. repetitions, $p<0.01$; see Methods). This pooling increased the number of significant cases from 15 to

878. 20 cells for subthreshold analysis, and 6 to 10 for spiking analysis (one of the additional cells was not

879. spiking). Once again, in congruence with the statistical analysis of Fig. 7, the largest proportion of

880. significant Surround-Only responses was found for the CP-ISO condition (CP-ISO: 12/37 cells, 32\%; CF-

881. ISO: 22\%; CP-CROSS; 8\%; RND-FULL: 11\%). The right column in Fig. 8 shows that the phase advance

882. effect is selective of the CP-ISO condition, where the mean Vm (top) responses, averaged across all 
883. "significant" cells ( $n=20$ for $V m$ ) indicate an average phase advance of $8 \mathrm{~ms}$ and a mean $V m$ response

884. integral increase of $31 \%$. A similar contextual phase shift is observed the spiking level (bottom).

885. Altogether, our intracellular results demonstrate that a build-up of anticipatory synaptic activity is

886. observed by sequentially recruiting local inducers co-aligned with the RF orientation axis in a

887. centripetal way. The efficiency with which the closest surround location is recruited impacts the

888. strength of the contextual modulation. In particular, the "Near" periphery recruitment of the

889. "cardinal" protocol points to a bias for CP-ISO AM flows along the RF preferred orientation axis,

890. superimposed over a non-specific facilitation component observed, at both Vm and Spiking levels for

891. all contextual stimulation conditions. Conversely, the more precise recruitment of the "Far" Surround,

892. achieved in the "radial" protocol, points to the selective control of the cortical gain by the CP-ISO

893. context. This specificity truly highlights a spatio-temporal requirement in Surround recruitment in

894. order to boost sensory responsiveness. This further emphasizes the critical need of spatial and

895. temporal synergy between each distal surround source synaptic impact with the synaptic feedforward 896. volley corresponding to the Center-Only drive (Fig. 1A).

897.

\section{4. Timing specificity in Predictive "filling-in" responses and Surround-then-Center interactions}

\section{4.1. Synaptic mechanisms involved in predictive filling-in responses ("radial" protocol)}

900. The time-course of the average contextual responses for cells that displayed significant responses

901. to the sole stimulation of the Surround, is compared in Fig. 9 between the CP-ISO AM SECTOR

902. configuration (red trace) and the three other flow configurations (light green for CF-ISO, gold for CP-

903. CROSS and blue for FULL RND-ISO). At the subthreshold level, the CP-ISO Vm response amplitude is

904. slightly amplified and its rising phase (measured at half height of the control peak response)

905. consistently leads by a $7 \mathrm{~ms}$ advance from the other conditions (top left panel, Fig. 9). At the spiking

906. level, peak values are undistinguishable, but a clear $15 \mathrm{~ms}$ advance only appears in spike initiation of 
bioRxiv preprint doi: https://doi.org/10.1101/2021.09.10.459746; this version posted September 11, 2021. The copyright holder for this preprint (which was not certified by peer review) is the author/funder. All rights reserved. No reuse allowed without permission.

Horizontal propagation of "network belief" in V1

20 August 2021

907. the CP-ISO condition. Additionally, the sustained depolarization observed for the CF-ISO condition

908. departing from the RF center is not translated at the spiking level, in contrast with the typical profile

909. observed for the same condition in the "cardinal" protocol (compare green and black waveforms in

910. the bottom left panel of Fig. 9, and filled histogram in the bottom panel and left part of Fig. 4).

911. Therefore, it appears that the "radial" protocol choice to avoid the "Near" periphery and restrain the

912. closest surround stimulation to the "Far" Surround enhances the specificity of the CP-ISO induced

913. facilitation, since phase advance and amplification of the Center-Only test response (black traces) are

914. only found for that specific condition. The averaging almost completely washed-out the non-specific

915. components found in the other AM conditions when using the "cardinal" protocol. Accordingly, in the

916. "radial” protocol, all other contextual response profiles (CF-ISO (green), CP-CROSS (gold) and RND-ISO

917. (blue)) are undistinguishable from Center-Only responses.

918.

919. Fig. 9 : Mechanistic analysis of the "Filling-in" contribution to contextual gain control.

920. Left, contextual gain control analysis, restricted to the population of cells $(n=12)$ showing significant "filling-in"

921. responses. Mean responses, averaged for each AM flow pattern (CP-ISO (red), CF-ISO (light green), CP-CROSS

922. (gold) and RND-ISO (blue)) are represented at the Vm (top panel) and spiking levels (bottom). Same color code as

923. in Fig. 1C. The selective latency advance and amplification of the subthreshold response induced specifically by

924. the CP-ISO condition (top, $n=12$ ) translate at the spiking level in cells displaying spiking activity (bottom, $n=7)$.

925. Right, comparison of the contextual time course of Surround-Only responses with the predicted contribution of

926. the Surround according to an additive model. Top, observed traces $(n=12)$; Bottom, linear predictors of the

927. Surround-Only responses obtained by subtracting the "Center-Only" trace from the "Surround-Then-Center"

928. response. The predicted profiles were calculated independently for each contextual condition (same color code).

929. The averaging process was extended to all cells showing a significant contextual change in latency and/or

930. response integral ( $n=15$, see criteria in Fig. 9). The expected occurrence of the response evoked by the

931. omitted Center stimulus is indicated by a grey shaded temporal window. The time abscissa of the peak of the

932. "Center-Only" stimulus response is indicated by a thin vertical bar.

933. 
934. The detailed comparison of the relative time-course of "filling-in" subthreshold response

935. contributions between the different AM flow conditions (Fig. 9, top right panel) shows that the

936. synaptic $(\mathrm{Vm})$ contribution of the "silent" periphery is the largest for the CP-ISO condition (red). Most

937. remarkably, it is temporally highly selective and peaks precisely at the expected time of occurrence of

938. the Center-Only response onset ("zero" relative time; see the dotted red trace in top right panel of Fig.

939. 6). The central issue addressed here is the temporal specificity of each purely contextual response and

940. their respective impact on the contextual gain control.

941. Indeed, this does not mean that other contextual flows are ineffective in modifying the

942. subthreshold baseline, such as the random (RND-FULL) configuration, which shows a depolarizing

943. plateau (blue trace) preceding the onset of the expected Center-Only response. This initial bump is

944. compatible with the cumulative invasion (across randomized strokes) of lateral activity and

945. corresponds to a non-null probability of stimulation by chance of the proximal periphery of the

946. subthreshold SRF. However, in contrast to the CP-ISO AM sequence, it collapses towards the baseline

947. before the expected onset of the Center-Only response. For the CF-ISO condition (green trace, top

948. right panel in Fig. 9), a mirror activation pattern regarding the Center-Only onset response is observed:

949. starting from rest, the transient CF-ISO Surround-Only contribution growth appears only after a delay

950. (30-35 ms), slightly after the peak of the Center-Only response had the test stimulus been presented,

951. before displaying a progressive depolarization decay lasting up to $250 \mathrm{~ms}$. This decay phase is

952. interpreted as indicative of distal input weakening and desynchronization, the further away from the

953. RF center (as expected from the stimulus design). In contrast, the CP-CROSS AM sequence synaptic

954. integration is almost ineffective (gold traces). The magnitude of its peak amplitude is several folds

955. lower than that observed in the "cardinal" protocol, reaching a peak value of only $8 \%$ of the Center-

956. Only peak response (compare gold traces in Fig. 3, 5A vs. Fig.s 5B and the top right panel in Fig. 9). This

957. argues once again for a "non-specific" component of the response, best seen in the "cardinal"

958. protocol, that crucially depends on whether the most proximal peripheral Gabor patch encroaches on

959. the SRF border or not. Note that, in spite of the fact that predictors of the Surround modulatory effect, 
bioRxiv preprint doi: https://doi.org/10.1101/2021.09.10.459746; this version posted September 11, 2021. The copyright holder for this preprint (which was not certified by peer review) is the author/funder. All rights reserved. No reuse allowed without permission.

Horizontal propagation of "network belief" in V1

20 August 2021

960. obtained by subtracting the feedforward response to the contextual response ("Surround-then-

961. Center" minus "Center-Only", shown in the bottom right panel of Figs. 6 and 9), could be computed

962. even in cells whose recordings did not show significant "Surround-Only" responses, a striking similarity

963. is apparent in the overall temporal profiles of top and bottom panels of Fig. 9. This isomorphism

964. globally supports on average a linear additive model of Surround and Center influences integration

965. (although some cells behave non-linearly, as shown in Fig. 6).

966. In summary, the CP-ISO condition is the only stimulation context where the time-course of the

967. Surround-Only contribution matches precisely the time-course required to interact with the feed-

968. forward activation window (shaded area, bottom right panel in Fig. 9) and define an effective "spiking

969. opportunity window". In contrast, in all other flow conditions, Surround-Only contribution is out of

970. sync with the feedforward drive and cannot exert mechanistically some form of additive gain control

971. of the test feedforward response. Crucially, the CP-ISO exclusivity of this precise time-localized

972. concentration in depolarizing activity power of purely contextual information actually explains the

973. selectivity of the contextual gain during complete sequences terminating in the RF center. This

974. extension in the temporal domain explains that those subthreshold synaptic responses "at the right

975. place, at the right time" can be viewed as a dynamic form of a neural correlate of "filling-in", intrinsic

976. to V1, propagating some kind of prediction of the spatio temporal profile of the next stimulus to come.

977. We therefore interpret those waves as indicative of a neural process of "anticipation" in the cortical

978. retinotopic region for which the "expected" visual feedforward synaptic volley is missing, indicative of

979. a running Bayesian estimate based on the recent stimuli statistics already presented in the Surround.

\section{4.2. Dependency of the spiking latency advance on the temporal phase between the Feedforward and Horizontal input waves ("cardinal" protocol)}

981. The timing constraints of our protocols have a dual source: i) the synaptic activation from the

982. surround displays a temporally constrained profile in latency basin (Fig. 2), and ii) the order of the

983. sequential stimulation (centripetal \& centrifugal) in the Surround (Fig. 4). In order to better quantify

984. these issues, the onset latencies of the postsynaptic potentials evoked independently in the Center- 
bioRxiv preprint doi: https://doi.org/10.1101/2021.09.10.459746; this version posted September 11, 2021. The copyright holder for this preprint (which was not certified by peer review) is the author/funder. All rights reserved. No reuse allowed without permission.

Horizontal propagation of "network belief" in V1

20 August 2021

985. Only and Surround-Only conditions were measured in the "cardinal" protocol and temporally

986. realigned on a common reference, defined as the time at which the Center-stimulus should have been

987. shown in the considered Surround-only 2-stroke AM sequence. The subtraction between the two input

988. onset latencies will be referred in the rest of the text as the phase between feedforward and

989. horizontal inputs. In spite of its weaker specificity, since the "cardinal" protocol almost systematically

990. induced significant responses for sequences limited to the surround and strong tonic responses at

991. both sub- and suprathreshold levels during complete sequences stimulating the center, we used the

992. measured onset latencies of this protocol to maximize the number of cells for this analysis. This latter

993. was however restricted to instances where both "Surround-Only" and "Surround-Then-Center"

994. responses were tested for each condition, in the same cells. The interest of such quantification is to

995. give an objective measure which allows the direct comparison of the effects of different associative

996. sequences realized for a given cell as well as across various cells (Fig. 10).

997.

998. Fig. 10 : Causal impact of the temporal phase between feedforward and horizontal input on the spiking latency change ("cardinal" protocol)

999. Left panel : Pooled data from the "radial" (brown), and "cardinal" (orange) protocols (this study) and from the

1000. "2-stroke" study by (29) (beige). Note that most apparent speed of horizontal propagation (ASHP) values,

1001. extracted from intracellular latency basin slopes with relative eccentricity, range between 0.05 and $0.60 \mathrm{~mm} / \mathrm{ms}$

1002. (with a peak between 0.10 and $0.30 \mathrm{~mm} / \mathrm{ms}$ ). Mirror histogram (pointing downwards) : pooled data from (26)

1003. using long bars (1D-mapping, blue) or sparse noise mapping (green). Right panel: For each individual cell

1004. recorded in the "cardinal" protocol, the latencies of Center-Only and Surround-Only Vm responses are measured

1005. and subtracted, defining the temporal phase between the "horizontal" and "feedforward (FF)" inputs. The

1006. scatter plot shows the relationship between the input phase and the resulting change in spiking latency of the

1007. recorded cell produced by the AM modulation (ordinate axis). Symbols for the centripetal conditions : " $x$ " in gold

1008. color (CP-CROSS-parallel) and " + " symbol in red color (CP-ISO-coaligned). Symbols for the centrifugal condition :

1009. “*” brown star (CF-CROSS-parallel) and green diamond (CF-CROSS-coaligned). In grey, bilinear fits. See Text for

1010. details. Note a significant reduction in spiking latency when the horizontal input is integrated post-synaptically

1011. ahead of the feedforward drive by 5-25 ms (phase advance, leftwards). The scatter plot data are projected on 
bioRxiv preprint doi: https://doi.org/10.1101/2021.09.10.459746; this version posted September 11, 2021. The copyright holder for this preprint (which was not certified by peer review) is the author/funder. All rights reserved. No reuse allowed without permission.

Horizontal propagation of "network belief" in V1

20 August 2021

1012. the x-axis (top row) or on the y-axis (right column) as Gaussian KDE fits. The color of the KDE distributions follow

1013. the convention of the "cardinal" visual stimulation protocol (Fig. 1B).

1014.

1015. When pooling all cells ( $n=23$ cells, 67 measures), the distribution of input phase relationships

1016. ranged from +25 ms of phase advance to -50 ms of phase lag between horizontal and feedforward

1017. input waves at the subthreshold level (abscissa in the bottom-right scatter plot of Fig. 10). As

1018. expected, a strong bias in the distribution was observed in favor of phase advance of the Surround

1019. input for the centripetal sequences (gold " $x$ " (CP-CROSS) and red " + " (CP-ISO) symbols) and delayed

1020. activation for the centrifugal sequences (brown "**" (CF-CROSS-parallel) and green diamond (CF-ISO-

1021. coaligned) symbols). At the spiking level, the latency shortening of the contextual (Surround + Center)

1022. output, compared to the Center-Only condition, appears crucially dependent on this input phase

1023. relationship: it was only observed for centripetal condition, i.e. when the Surround response onset

1024. (the horizontal input), preceded in time the Center-only response onset (the feedforward (FF) input).

1025. The intercept of the grey bi-linear fit, shown in the scatter plot of Fig. 10, has been optimized by two

1026. half-regression lines independently of the stimulus conditions (taking all the data points together). The

1027. correlation optimization results in a partition of the data cloud in two regions: one (on the left side)

1028. where the horizontal input lags in phase the feedforward drive, and for which no significant trend is

1029. observed; the other (on the right side), where the horizontal input is integrated postsynaptically ahead

1030. of the feedforward drive by 5-25 ms. In the ordinate domain, a significant reduction in spiking latency

1031. is observed (latency advance pointing upwards on the y-axis), reflecting in an almost linear way the

1032. phase advance of the synaptic echo of the horizontal input relative to that of the feedforward drive

1033. ("0" abscissa).

1034. The Gaussian kernel density estimator (KDE) of the input phase distribution (along the $\mathrm{x}$-axis,

1035. above the scatter plot) clearly shows that the depolarizing input wave evoked by a collinear pair of

1036. stimuli flashed in a centripetal sequence in the "Near" periphery (CP-ISO, in red) was arriving on

1037. average earlier ( $17 \mathrm{~ms}$ from the scatter plot projections; $9 \mathrm{~ms}$ for paired data) than when the stimuli 
1038. were oriented orthogonally to the width axis path (CP-CROSS, in gold). This phase difference at the

1039. subthreshold level was significant in $65 \%$ of the cells where both Surround-Only and Surround-then-

1040. center conditions were tested for CP-CROSS and CP-ISO conditions (Z-test from resting state, $\mathrm{p}<0.05$ ).

1041. Note that, in some cells, the latency advance at both Vm and Spiking levels of the CP-CROSS

1042. stimulation (gold symbols) is non-null. However, the average $9 \mathrm{~ms}$ difference in subthreshold

1043. responses of CP-ISO and CP-CROSS conditions of the "cardinal" protocol corresponds to the $8 \mathrm{~ms}$

1044. latency advance (between CP-ISO and Center-Only response), found for the CP-ISO significant

1045. subpopulation of "significant" cells in the "radial" protocol. Note that, for this precise contingent, the

1046. latency advance component of the CP-CROSS response was undistinguishable from the reference (Figs

1047. 7, 8 and 9).

1048. Only centripetal collinear CP-ISO sequences could induce significant shortening in output spiking

1049. latency, by an advance as large as $30 \mathrm{~ms}$. As shown by the KDE distribution on the $y$-axis (right of the

1050. scatter plot), the median latency reduction observed for the collinear axis during a centripetal

1051. stimulation (red line on the shaded red envelope) was $11 \mathrm{~ms}$. In the other conditions, either

1052. centrifugal or centripetal "parallel" along the RF width axis, no significant latency advance could be

1053. seen: the three other distribution fits are all centered on a null latency change. At the spiking level, the

1054. median difference between the onset latency of CP- and CF-conditions is of $13 \mathrm{~ms}$ (respectively, red

1055. and green lines).

1056. Although a significant difference in the distribution of surround modulation of the depolarizing

1057. and spiking responses is found between "parallel" and "collinear" conditions, no significant difference

1058. other than latency change could be revealed when comparing the centripetal vs. centrifugal conditions

1059. of the "cardinal" protocol. On average, the integral values of centripetal depolarizing and spiking

1060. responses (measured over a $100 \mathrm{~ms}$ window) were respectively 1.1 and 1.2 times larger than that of

1061. centrifugal responses. At the Vm level, this modest change is likely to be due to the broad duration of

1062. surround responses $(130 \mathrm{~ms})$ because of the strong response evoked by the closest GP in the "Near"

1063. periphery and to the existence of multiple phase relations between lateral and feedforward input 
bioRxiv preprint doi: https://doi.org/10.1101/2021.09.10.459746; this version posted September 11, 2021. The copyright holder for this preprint (which was not certified by peer review) is the author/funder. All rights reserved. No reuse allowed without permission.

Horizontal propagation of "network belief" in V1

20 August 2021

1064. during the time course of the associative process. This finding is indeed replicated in the "radial"

1065. protocol, with an average facilitation of the center-only response integral difference of only $10 \%$

1066. between CP- and CF-ISO responses (Fig. 8 and Fig. 9, top right panel). At the spiking level, this modest

1067. difference is likely linked to the shorter duration of stimulation (16 ms) used in the "cardinal" protocol,

1068. opposed to the average $30 \pm 9$ ms stroke duration of each GP used in the "radial" one, for which

1069. facilitation of the center-only integral response was not observed, probably because of the refractory

1070. period of cells that were already stimulated in the center of their receptive field.

\section{4.3. Synergy depends on the relative match between apparent motion and intracortical horizontal propagation speed}

1072. A subtle difference between the two types of protocols is that the AM speeds used in the "radial"

1073. protocol depended on the GP duration and on the size of the SRF and not only on the size of the MDF

1074. (as this was the case for the "cardinal" case). This finer speed tuning was adjusted to the

1075. characteristics of each individual cell since the response latency and strength of evoked responses

1076. both depend on the stroke duration and the distance between strokes. For the 37 cells of the "radial"

1077. protocol, the average visual stimulation speed was of $189 \pm 47 \%$ sec, (ranging from 72 to $312 \%$ ),

1078. hence below the average speed of $329 \pm 101 \%$ (range $175-500 \%$ s) used in the "cardinal" protocol.

1079. Note that despite this difference, the global ASHP distributions reported in the "radial" and "cardinal"

1080. protocols overlap closely with the overall range of distributions reported in (26) and (29) (mirror

1081. histograms in left panel of Fig. 10). A detailed comparison shows that the inferred speed of laterally

1082. conveyed activity is slightly accelerated for "dynamic" sequences of AM flows recruiting the periphery

1083. (top of the histogram) than for "static" flashed stimuli measures (bottom), peaking respectively at

1084. 0.175 and $0.125 \mathrm{~mm} / \mathrm{ms}$. Those results point to the notion that the speed of laterally conveyed activity

1085. is not completely hard-wired but depends partly on the type of visual stimulus used. Therefore, higher

1086. levels of spatio-temporal coherence along the motion path slightly increases the speed of laterally

1087. mediated activity, leading most likely to a cumulative shortening of the response onset latency at each

1088. updated locus of stimulation of the AM path in the cortex, a phenomenology already reproduced in 
bioRxiv preprint doi: https://doi.org/10.1101/2021.09.10.459746; this version posted September 11, 2021. The copyright holder for this preprint (which was not certified by peer review) is the author/funder. All rights reserved. No reuse allowed without permission.

Horizontal propagation of "network belief" in V1

20 August 2021

1089. simulations of nested horizontal propagation (47).

1090. Functionally, a critical prediction of our "working hypothesis" is that the visibility of the lateral

1091. contextual modulation of the feedforward input should therefore increase when the speed of the AM-

1092. evoked wave in cortex grows closer to horizontal propagation speed. Focusing on the SECTOR

1093. configuration of the "radial" protocol, we studied more in depth the impact of the CP-ISO AM kinetic

1094. effects on synaptic integration in V1 in a subset of twelve cells, by comparing, in each of these cells,

1095. the impact of AM sequences of various speeds. The speed of lateral connectivity recruitment was

1096. controlled in a graded way by introducing temporal delays between GPs strokes (detailed in the

1097. chronograms in the bottom panel of Fig. 11). Each cell "optimal" speed of periphery recruitment was

1098. taken as the $100 \%$ reference speed, and was lowered to $70 \%, 50 \%$ and $30 \%$ in a pseudo random

1099. fashion in the same cell.

1100.

1101. Fig.11 : Dependency of the Contextual effect on the speed of the AM flow :

1102. Bottom, the chronograms depict the 4 inter-stroke interval conditions used in each cell to probe the temporal

1103. dependency of the interaction between the horizontal and the feedforward synaptic waves (color code reddens

1104. with speed value). The exact value of the "optimal" reference speed (100\%) is defined on a cell-by-cell basis.

1105. Mean speed values, averaged across cells $(n=12)$, are given left of each chronogram. Top, population averages

1106. for the CP-ISO SECTOR condition. All individual responses are realigned with the Center-Only response onset. The

1107. overlaid waveforms, averaged across cells, allow the comparison of the time-course of the response for the

1108. optimal speed value (red) with that observed for various proportional reductions of the AM flow speed (from top

1109. to bottom: $30 \%$ (dark orange), 50\% (orange) and 70\% (light orange)). The contextual response modulation

1110. amplitude decreases proportionally to the AM speed reduction from its optimal value.

1111.

1112. The comparison of the average contextual PSP response profiles of this subpopulation for

1113. incremental AM speeds (30\%, 50\%, $70 \%$ and $100 \%$ of the "optimal" value) highlights a cumulative

1114. speed-dependent anticipatory recruitment of the periphery, with a bumpy stairway-like profile

1115. preceding the main response peak (top panel in Fig. 11). Crucially, the overlay of response profiles for 
bioRxiv preprint doi: https://doi.org/10.1101/2021.09.10.459746; this version posted September 11, 2021. The copyright holder for this preprint (which was not certified by peer review) is the author/funder. All rights reserved. No reuse allowed without permission.

1116. different speeds suggests a progressive merging of lateral inputs impinging on the central target RF,

1117. from weaker amplitude and asynchronous early responses at lower speed to larger amplitude and

1118. synchronous "in-phase"-summation with the feedforward drive (Center-Only, black curve in Fig. 11)

1119. when approaching the "optimal" AM speed. As speed value grows (from $30 \%$ (light orange) to $100 \%$

1120. (red) of the optimal speed), lateral influence gets progressively synchronized when it reaches the RF

1121. center. This is attested by the presence of dissociated bumps (preceding the Center response) at

1122. slower speed, which likely reflects sequences of asynchronous activation by the distal surround

1123. sources. In contrast, at the "optimal" speed, the precise time-concentration increase in depolarizing

1124. activity power enables a more efficient interaction with the center-only response, thereby boosting in

1125. a causal way the feedforward PSP. This result clearly emphasizes the crucial timing of synaptic

1126. recruitment in the Surround, which controls the emergence of synergy between long-distance (orange

1127. arrows in Fig. 1A) and cascades of shorter neighbor-to-neighbor links (grey arrows in Fig. 1A) along the

1128. AM path. We conclude that "in-phase" summation of lateral inputs with the predicted time of the

1129. feedforward drive produces the shortening of latency and amplitude amplification reported during CP-

1130. ISO flow at high AM speed.

1131. Altogether, our results show that facilitatory contextual control of V1 responses is optimized

1132. along the orientation preference axis when the global motion speed lies within the range of visual flow

1133. speed predicted from the apparent conduction velocity of horizontal connectivity. For slower motion,

1134. the horizontally-driven facilitation effect disappears and stimuli cross-oriented to the motion path,

1135. presented across the width axis, remain the best drivers of cortical cells. We conclude that the axial

1136. preference of $\mathrm{V} 1$ cells shifts abruptly by an angle of $90^{\circ}$ when the motion speed switches from 2-20

1137. $\%$ s to $100-250 \%$ s.

1138. In summary, the spatial congruence of the elements regarding the preferred orientation axis, the

1139. spatio-temporal coherence of the flows regarding the RF center (centripetal), the speed of recruitment

1140. of the periphery and the precise timing selectivity of Surround-only responses, all suggest that some

1141. form of peripheral subthreshold "prediction" is generated internally in V1, to interact, within the 
bioRxiv preprint doi: https://doi.org/10.1101/2021.09.10.459746; this version posted September 11, 2021. The copyright holder for this preprint (which was not certified by peer review) is the author/funder. All rights reserved. No reuse allowed without permission.

Horizontal propagation of "network belief" in V1

20 August 2021

1142. proper spatio-temporal window, with the feedforward Center-Only synaptic volley. These highly

1143. selective features justify, to our view, the qualification for the existence of neural correlate of

1144. "predictive filling-in" in V1, to be distinguished from a non-specific anticipatory depolarizing spread.

1145. 
bioRxiv preprint doi: https://doi.org/10.1101/2021.09.10.459746; this version posted September 11, 2021. The copyright holder for this preprint (which was not certified by peer review) is the author/funder. All rights reserved. No reuse allowed without permission.

\section{Discussion}

1147. Most studies of contextual modulation in the early visual system are based on synchronous

1148. Surround and Center stimulation, pointing to mixed suppressive and facilitatory effects $(13,14,48-53)$.

1149. We document here a dynamic regime of asynchronous Surround-Center activations, of specific speed

1150. and spatial anisotropy, which, under precise timing and co-alignment conditions, reveals a facilitatory

1151. form of contextual cortical transfer function control. This effect, which links global motion direction

1152. and local orientation, is strong enough in the anesthetized brain to shorten spiking response latency

1153. by a few tens of milliseconds and shift the axial preference of V1 cells for saccadic-like speed, favoring

1154. contour integration along the orientation preference axis.

\section{1. Orientation anisotropy, Motion streaks and Speed sensitivity}

1156. The dependency on co-alignment and motion along the RF preferred orientation axis reported

1157. here correlates with the anatomical bias for iso-oriented long range connections described in higher

1158. mammalian visual cortex (cat: (54-56); tree shrew: (57-59); macaque : $(60,61)$; squirrel monkey : (62)).

1159. At the functional level, it fits with the concepts of the perceptual "Association Field" in humans (4),

1160. the neural "facilitation field" in the behaving NHP $(11,63)$ and the synaptic "Dynamic Association

1161. Field" in cat V1 (29). In terms of efficiency, the requirement of maximizing the spatio-temporal synergy

1162. from the Surround corroborates with the recruitment impact of long-distance iso-tuned interactions

1163. visualized with imaging techniques at the mesoscopic level (40).

1164. Importantly in V1 neurons, the integration axis (the RF preferred orientation) for which the

1165. strongest responsiveness modulation by fast $\left(150-250^{\circ} / \mathrm{s}\right)$ apparent motion is observed, is "orthogonal"

1166. to the preferred directional axis of V1 neurons (the RF width axis) when probed with slowly moving

1167. bars and full field gratings. Classically, area 17 (V1) receptive fields are best activated by slow motion

1168. across the RF width axis (1-2\% in (46); 3-10\% in (64), but quantitative studies however reveal a zoo

1169. of speed tuning selectivities in V1 ((65-67); review in (68)). Among these, "parallel motion direction

1170. selective" neurons could provide a potential substrate of the effects reported here. However such 
1171. trigger features are classically found in higher visual cortical areas dedicated to global motion (type II

1172. MT cells (69)), but not in V1. In contrast, Geisler and colleagues hypothetized, then described, a

1173. specific class of cells in V1 responding to Gaussian blobs, named "streak" detectors, oriented in the

1174. direction of motion $(70,71)$. To account for disambiguation of motion direction, Geisler introduced an

1175. hypothetical multiplier circuit between direction selective and parallel detectors, whose existence is

1176. still to be demonstrated (70). Realistic estimates for the speed threshold above which "motion streak"

1177. operates point to medium range values, around $20-60 \% \mathrm{~s}$ for cat and $10-20 \% \mathrm{~s}$ for monkey (inferred

1178. from (72)). This "streak" effect, however, depends on the test stimulus, i.e. small isotropic Gaussian

1179. blobs, critical to avoid recruiting side-inhibition, and is absent for oriented plaid patterns (71). Other

1180. studies reported related findings: Orban and colleagues showed that spot directional tuning shift by

1181. $60-90^{\circ}$ above a speed threshold, whereas bar tuning remained invariant $(68,73)$. Intrinsic optical

1182. imaging experiments - combining textures tiled with small bars and independent motion components

1183. conclude to a complex dependency of orientation domains recruitment on local motif length and

1184. motion speed (74). Similarly, $90^{\circ}$ shifts in orientation domains were described for moving dot noise

1185. above $14.5 \% \mathrm{~s}$ in cat area $17(75)$.

1186. In contrast, the $90^{\circ}$ shift in axial motion sensitivity reported here is found at much faster

1187. (saccadic-like) AM speeds $\left(>50^{\circ} / \mathrm{s}\right)$, and rely on the same local inducer (oriented Gabor) shifted in

1188. space and time. It is the precise time-lagged recruitment of Surround-then-Center synaptic inputs, that

1189. conditions the contextual amplification and the phase advance of the Center response. Both the

1190. binding specificity of the local (orientation) and global (motion axis) features of the AM flow, on the

1191. one hand, and the match between the optimal AM speed and ASHP values, on the other hand, support

1192. the view that the main player is long-range horizontal connectivity intrinsic to V1.

\section{2. Novelty of the study}

1194. The novelty of our findings is several fold : 1) these lateral diffusion effects recorded in the

1195. anesthetized, hence non-attentive, animal are likely to be intrinsic to V1 and do not require behavioral 
bioRxiv preprint doi: https://doi.org/10.1101/2021.09.10.459746; this version posted September 11, 2021. The copyright holder for this preprint (which was not certified by peer review) is the author/funder. All rights reserved. No reuse allowed without permission.

Horizontal propagation of "network belief" in V1

20 August 2021

1196. attention and top-down control from higher cortical areas (weakened by anesthesia) ; 2) they depend

1197. on the spatiotemporal coherence of the AM sequence and grow with flow speed until matching the

1198. apparent speed of horizontal propagation (ASHP) in V1;3) in that range of speed, they produce a shift

1199. by $90^{\circ}$ of axial motion sensitivity, which becomes co-aligned the preferred orientation RF axis; 4) they

1200. provide evidence for an internally-generated propagation process, binding local (orientation and

1201. position) and global (motion and direction) features; 5 ) they constitute a plausible neural substrate of

1202. psychophysical effects in humans reporting a perceptual bias in speed estimate for collinear motion

1203. (76).

\section{2.1. Intra-V1 horizontal diffusion vs. cortico-cortical feedback}

1205. Conventional models of visual cortical processing assume that complex computations, such as

1206. motion direction disambiguation, are encoded only at higher levels in the visual cortical area hierarchy,

1207. and are absent in $\mathrm{V} 1(9,10,77-81))$. Most electrophysiological studies in the behaving primate point to

1208. the late retroaction in V1 of a top-down signal gated by attention (82-85). They attribute the delay of

1209. several tens of milliseconds of the change in firing of V1 cells after response onset, to the late action of

1210. the cortico-cortical feedback (86-90), rather than to the slow horizontal propagation in V1. However,

1211. puzzlingly enough, axonal feedback transmission from higher areas is $10-100$ times faster than intrinsic

1212. diffusion in V1 (see also (91) for the parallel recruitment of dual lateral-feedback kinetics).

1213. The challenging view presented here is that, as early as V1, horizontal connections play already an

1214. important role in the early perception of retinal flow and global motion. The visibility and spatial

1215. spread of their functional dominance in cats \& ferrets vs non-human primates (NHP) may reflect

1216. species-specific differences in retino-cortical magnification factor. Horizontal axon length (4-8 $\mathrm{mm})$ is

1217. the same across species, but covers up to $8^{\circ}$ of visual angle in cat-ferret (cat : $(54,55,92)$; ferret : $(59)$

1218. whereas it remains within a radius of 1.5 hypercolumn $\left(2-3^{\circ}\right)$ in primates $(93)$, which matches the

1219. spatial extent of collinear facilitation observed in human psychophysics (42, 94-96).

1220. The multi-stroke AM protocols ("cardinal" and "radial") were both designed to promote the in-

1221. phase synchronization of the synaptic impacts produced by the sequential stimulation of each GP 
bioRxiv preprint doi: https://doi.org/10.1101/2021.09.10.459746; this version posted September 11, 2021. The copyright holder for this preprint (which was not certified by peer review) is the author/funder. All rights reserved. No reuse allowed without permission.

1222. flashed along the motion path with the central feedforward drive (orange arrows in Fig. 1A). Our

1223. prediction was that this synchronization would be optimized for a flow speed in the range of the

1224. apparent propagation speed of horizontal connectivity (ASHP in (26) and has been confirmed by the

1225. speed-dependency tuning study reported here (see Results). However, the variability of the

1226. intracellular anticipatory Vm profile across cells suggests that other synaptic recruitment paths

1227. are likely to contribute, in particular the neighbor-to-neighbor links (grey arrows in Fig. 1A). It is thus

1228. likely that the "horizontal" drive efficiency results from a combination of the asynchronous visual

1229. activation of two classes of synaptic sources: 1) long-range monosynaptic inputs recruited at different

1230. eccentricities, whose distributed and delayed impact is integrated in phase by the target cell; 2)

1231. polysynaptic short-range input, reflecting chains of next-to-next neighbor activation (studied in our

1232. previous 2-stroke AM (29)). This second effect is amplified by the re-ignition process, imposed at each

1233. GP locus by the next feedforward input recruited on the apparent motion trajectory. Note that this

1234. rolling wave effect is not seen for single stroke stimuli, since, otherwise, the spiking discharge field of

1235. the recorded cell would extend far beyond the spatial region defined by the core thalamo-cortical

1236. projection.

1237. Since the reverberation delay imposed by cortico-cortical loops is in the order of a few

1238. milliseconds only (97), it is plausible that most long-range interactions in the primate may operate by

1239. nested polysynaptic feedback afferents from higher cortical areas operating through chains of

1240. neighbor-to-neighbor lateral relays, whereas in the cat and ferret, a substantial part would remain

1241. encapsulated monosynaptically within V1 (7). Our results may provide new insights on the

1242. evolutionary complexification of cortico-cortical computation. They could also spark interest in Al and

1243. deep learning in artificial vision, where the diffusion effects of horizontal connectivity in a single V1-

1244. like terminal layer can be confronted with the backpropagation of "network belief" implemented by

1245. nested feedback connections in a multi-layer hierarchical cortical-like architecture (98).

\section{2.2. Dependency on spatiotemporal coherence and visual flow speed}

1247. Since 2-stroke AM provided evidence mostly for subthreshold synaptic amplification (29), we 
1248. aimed here at promoting the efficiency of centripetal AM CP-ISO sequences to produce a spiking

1249. discharge, by increasing the number of elementary strokes in the AM sequence, from 3 to 6 from the

1250. "cardinal" to the "radial" protocols. In the "radial" protocol, the comparison between FULL-RND,

1251. SECTOR-RND (data not shown), and SECTOR-CP-ISO conditions demonstrates that the boosting of

1252. sensory responsiveness grows with the level of spatio-temporal coherence. The speed dependency

1253. demonstrates unambiguously that the contextual control of the cortical transfer function is maximized

1254. when a causal coherence is reached, i.e. when the horizontal wave predicts the arrival of the

1255. feedforward wave.

1256. The optimal range of speed $\left(150-250^{\circ} / \mathrm{s}\right)$ reported here contrasts with a recent study of

1257. anticipatory responses in the fixating monkey, best seen for stimuli cross-oriented to the motion path,

1258. in continuous slow motion across the RF width axis $\left(6.6^{\circ}-13.2^{\circ} / \mathrm{s}\right)(99)$. The authors propose a complex mechanism, based on the interplay of the "feedforward" trace imposed by the continuous motion of

1259. the stimulus and a medium range isotropic horizontal propagation wave. This activity bump still allows

1260. a suboptimal slight interaction with the feedforward drive when it propagates faster than the stimulus.

1261. Their interplay would cause a pre-activation of the cells, facilitating in turn the feedforward

1262. reactivation. This proposed mechanism is highly dependent on the retino-cortical magnification factor

1263. and does not account for the global dynamics of the anticipatory wave for distances beyond

1264. monosynaptic connection lateral extent. The propagation speed across long trajectories $\left(>3^{\circ}\right.$ from the

1265. Spiking discharge field center), well beyond the spatial extent of monosynaptic links observed in NHP

1266. $\left(<2^{\circ}\right)$, is indeed ten times slower $(<=0.02 \mathrm{~mm} / \mathrm{ms})$ than the apparent speed of horizontal propagation

1267. (0.05-0.50 mm/ms; see $(26,32))$. The global kinetics is more in line with a slower "rolling wave"

1268. diffusion mechanism (see also in vitro (100)). The speed range of this anticipatory wave reported in

1269. awake macaque $\left(<15^{\circ} / \mathrm{s}\right)$ is lower than in the anesthetized cat area $17\left(15-40^{\circ} / \mathrm{s}\right.$ in (39)), who showed

1270. that a moving square is more rapidly processed than a flashed one. These different effects share some

1271. similarities with those reported here, but differ significantly in their speed range (by a 10-20 fold) and

1272. preferred axial sensitivities (CROSS-parallel vs ISO-Collinear). Furthermore, they do not require 
1273. collinearity, an hallmark of horizontally-mediated V1 computation.

\section{2.3. Binding of global and local features in V1}

1275. The current consensus concerning cortical correlates of low-level perception mixes hierarchical

1276. processing and cortico-cortical recurrency : V1 plays a pivotal role in synchronization dynamics back

1277. and forth with high-order cortical areas $(16,101)$. Intra-V1 synchronization and inhibitory interactions

1278. are hypothetized to interact through a form of "competitive coding", before the output is further

1279. processed downstream in the cortical hierarchy. A non-exclusive alternative is that reverberating

1280. activity in V1 already concurs to non-attentive "pop-out" perception. We propose that horizontal

1281. connections, intrinsic to $\mathrm{V} 1$, are instrumental to the neural implementation of low-level perceptual

1282. Gestalt laws, in parallel or before information is broadcasted to the mosaic of higher cortical areas.

1283. Our data suggest that, in Gestalt-like configurations, they propagate some kind of "network belief"

1284. (built-up on past activity ignition) to the connex V1 neighborhood, the most likely to be next

1285. stimulated (102).

1286. Remarkably enough, the "prediction" wave, seen for "Surround-Only" AM CP-ISO sequences,

1287. binds local information acquired along the motion path. This observation, although recorded in the

1288. anesthetized brain, globally fits with Bayesian theoretical frameworks, where omission of the next-to-

1289. come stimulus in repetitive and predictive activation sequences unmasks an intracortically generated

1290. "expectation" signal mediated by horizontal connectivity. This finding has to be distinguished from the

1291. classical "predictive coding" schema (103), where what is propagated is the "residual error" from the

1292. expectation generated in higher cortical areas rather than the diffusion of the network belief context

1293. (98). At optimized speed, the self-generated "Surround-Only" wave is shown here to exhibit the same

1294. timing as the FF input onset evoked by the next stimulus to come, had it been presented. More

1295. exploration, outside the scope of this paper, would be needed to test how center-surround interaction

1296. is affected by the contrast (low/high) and the orientation (same/mismatch) of the test Center stimulus, 1297. and how possible sub-linearities can be interpreted in the context of the principle of redundancy

1298. reduction framework (104). 
bioRxiv preprint doi: https://doi.org/10.1101/2021.09.10.459746; this version posted September 11, 2021. The copyright holder for this preprint (which was not certified by peer review) is the author/funder. All rights reserved. No reuse allowed without permission.

Horizontal propagation of "network belief" in V1

20 August 2021

1299. Our results suggest that V1 may solve the motion extrapolation problem just through selective

1300. internal diffusion. Our interpretation is supported by theoretical studies of motion extrapolation

1301. (ability to continue to predict accurately the position/speed of a moving object in the sudden absence

1302. of visual input): Kaplan and colleagues implemented a realistic spiking recurrent neural network

1303. simulation of V1/MT (105), and studied cortical dynamics relaxation, when the coherent motion of a

1304. dot was abruptly interrupted by a "blank" period for three different lateral connectivity distributions

1305. (random, isotropic and anisotropic). The main finding, in agreement with this study, was that

1306. anisotropy was required to allow an efficient diffusion of motion information and achieve accurate

1307. motion/position extrapolation in the absence of FF validation (Fig. 4 in (105)).

\section{2.4. A neural correlate of perceptual bias in speed estimate for collinear motion}

1309. Our electrophysiological findings constitute a potential correlate, as early as V1, of the human

1310. psychophysical speed-up bias in motion flow integration $(47,76)$. These previous psychophysical and

1311. computational studies from our lab showed that collinear AM sequences - composed of co-aligned

1312. ISO-Gabor patches along the global motion axis - are perceived by humans as moving "faster" than AM

1313. sequences composed by "parallel" CROSS-oriented elements, of the same exact physical speed. The

1314. speed bias decreased as the angle between the local GP inducer and the global motion axis increased.

1315. This bias was highly dependent of the orientation selectivity of the inducer and disappeared for blobs,

1316. which suggests a cortical origin. All reported features show a close link with the synaptic association

1317. field of V1 neurons (29) and the reported data.

1318. Crucially, the speed, for which the speed-up effect is the strongest in humans, peaks at $64 \%$.

1319. Assuming a parafoveal retino-cortical magnification factor ratio of 3 between human and cat (human:

1320. (106); cat: (107)), the optimal speed-up range of $40-96^{\circ} / \mathrm{s}$ seen in human would correspond to 120 -

1321. $288^{\circ} / \mathrm{s}$ in cat, which fits remarkably well the $100-312^{\circ} / \mathrm{s}$ reported here. The $64^{\circ} / \mathrm{s}$ optimal velocity for

1322. humans corresponds for the cat to a speed of $192 \%$ on the retina, and an apparent propagation

1323. speed of $0.19 \mathrm{~mm} / \mathrm{ms}$ in cortex, in agreement with the inference made from our intracellular 
1324. recordings. Most remarkably, the boosting mechanism revealed in this study confirms the prediction

1325. of an earlier computational model of our laboratory (47), which provided a simple conceptual

1326. framework where synaptic summation between feedforward and lateral AM inputs results in

1327. amplification and latency advance in V1 spiking responses.

1328. Our intracellular study allows to go one step beyond this spike-based modeling. In (47), the

1329. composite synaptic summation is linear and the non-linearity of the functional effect is simply due to

1330. that of spike initiation. However, the intracellular study of the timing dependency of the association

1331. process suggests a non-linearity in the interaction process between feedforward (FF) and horizontal

1332. input, which becomes supra-linear when FF input lagged by more than 5.5 ms the contextual signal

1333. (Fig. $7 \mathrm{C}$ in (29); see also here Fig. 10, right pane)). It remains plausible that a minimal integration time

1334. is needed to recruit non-linear voltage-dependent mechanisms recruited by horizontal connectivity,

1335. such as the persistent sodium current or NMDA receptor activation $(25,108)$.

\section{3. Concluding statements}

1337. In conclusion, the view presented here differs significantly from classical studies/models of top-

1338. down gain control of V1 processing which ignore the contribution per se of horizontal connectivity (11, $15,16)$ or attribute the late enhancement of visual responses in $V 1$ to the exclusive feedback from

1339. higher cortical areas (83). We do not argue here against the consensus that top-down signals are

1340. required, during attentive wakefulness, to express and/or amplify an integrative process intrinsic to V1.

1341. However, our recent demonstration of a "synaptic association field" in the anesthetized preparation

1342. demonstrates a preexisting structural bias intrinsic to V1, that could constitute the synaptic footprint

1343. of the neural architecture needed to implement Gestalt laws (29). This finding is further strengthened

1344. by a multiscale imaging study (40), suggesting that a critical threshold of spatial summation has to be

1345. trespassed in order to make these long-range interactions detectable, while temporal synergy

1346. conditions the effective binding of those spatial interactions (39). A likely interpretation is that some

1347. kind of resonance regime, such as produced here by the multiple stroke reafferent drive, is needed in 
1348. the anesthetized state to propagate at the spiking level a functional bias already present in the

1349. subthreshold impact of horizontal connectivity.

1350. The novel conceptual focus here is on the temporal features of horizontal propagation and on

1351. intra-V1 broadcasting. State-of-the-art modeling generally ignores the propagation distance-

1352. dependent delay component whose impact compromises greatly the analytical solving of mean-field

1353. equations (109); but see (110)). Interestingly, recent large-scale network simulations with

1354. topographically-organized horizontal monosynaptic connectivity and distance-dependent axonal

1355. conduction delays $(0.10-0.60 \mathrm{~m} / \mathrm{s})$ demonstrates the consistency of spontaneous subthreshold waves

1356. with the asynchronous-irregular regime, defining a sparse-wave regime, more in line with a realistic

1357. view of a functional V1 (36). Although our results interpretation seems in agreement with some of the

1358. computational literature on predictive coding applied to contour-through-motion extrapolation and

1359. trajectory extraction, they still remain to be confronted with alternative models which do not require

1360. propagation of a prediction. Other classes of probabilistic and static models (71) do not depend on

1361. propagation at finite speed and apply primarily to isotropic stimuli which are known to be suboptimal

1362. to activate V1.

1363. Overall, our recordings suggest that fast collinear flow facilitates the refinement of anisotropy

1364. integration, distributed over the mesoscopic representation of trajectories across the "horizontal"

1365. layer plane of V1. The Surround-only CP-ISO anticipatory wave, captured "at the right position, at the

1366. right time" in a subset of our intracellular recordings, is indicative of a progressive topological

1367. refinement of activity diffusion in cortical space. In cat visual cortex, using annular stimuli and voltage

1368. sensitive dye imaging, Chavane et al reported correlates of "filling-in" responses (40), which fit with

1369. our subthreshold membrane potential recordings. Using intrinsic optical imaging (which might reflect

1370. more spiking than subthreshold activity), Chisum et al reported in the anesthetized tree shrew, that

1371. ISO-oriented collinear Gabor arrays flashed simultaneously did enhance locally V1 sensory

1372. responsiveness (111). This latter study, which used a static input pattern of 2 second duration,

1373. activating simultaneously all the Surround sites, detected some activity strengthening in the 
1374. areas directly (FF) activated by co-aligned local inducers, but failed to observe any spatial expansion of

1375. lateral activity in the cortical regions representing stimulus gaps. In contrast, our study predicts that

1376. the dynamic apparent motion animation of the same array, activating in succession the co-aligned

1377. local oriented inducers, would generate a continuous anisotropic propagation wave, most visible for

1378. AM speed faster than $50^{\circ} / \mathrm{s}$. This diffusion process would, in turn, fill the gaps between local

1379. feedforward activation zones and promote the extraction of a motion trajectory riding on an

1380. expanding collinear contour. Evidence in V1 for an expansive "filling-in" process during apparent

1381. motion finds some support in fMRI imaging studies in humans (112). It could be tested spatio-

1382. temporally with more precision in cat and NHP, using voltage sensitive dye imaging and fast AM

1383. sequences.

1384. Irrespectively of the species-dependency of the visual spatial coverage of horizontal connections

1385. ( $7-8^{\circ}$ in cats vs $1-2^{\circ}$ in NHPs, in spite of similar axonal length), multiple stroke AM sequences appear

1386. best suited to dynamically recruit overlapping "lateral connectional fields" along the motion trajectory

1387. (90). The optimal separation in the visual field between the GP inducers might however be still

1388. species-specific, since the orientation selectivity of the horizontal facilitation process seems best

1389. prominent when co-stimulating the "Far" surround of GP-driven RFs. This intriguing propagation of

1390. self-generated and propagated prediction at an early stage of visual cortical processing may underlie a

1391. more profound functional role. It also suggests a possible interpretation of the peculiar geometry of

1392. saccadic scanpaths of human observers during the viewing of other human faces. Indeed, Alfred

1393. Lioukianovitch Yarbus long ago reported a striking similarity between the topographic layout of the

1394. saccadic scanpaths with the most salient collinear contours outlining the perceptual skeleton of the

1395. face viewed by the observer $((27,113,114)$; review in $(115))$.

1396. 


\section{Acknowledgments :}

1398. We thank Vincent Bringuier, Frédéric Chavane, Jean Lorenceau, Peggy Seriès and Florian Gérard-

1399. Mercier for their past collaborative contribution to the concept of the "synaptic association

1400. field". We are indebted to Frédéric Chavane, who participated in his PhD work to some of the

1401. early experiments. We thank Gérard Sadoc for the in-house development of visual stimulation

1402. and data acquisition software (Elphy ${ }^{\mathrm{TM}}$ ), Kirsty Grant and Guillaume Hucher for histology and

1403. Aurélie Daret for her support during experimental surgery. 


\section{References :}

1. Wertheimer M. Experimentelle Studien über das Sehen von Bewegung [Habilitation Frankfurt]. Leipzig: J. A. Barth; 1912. Zeitschrift für Psychologie und Physiologie der Sinnesorgane, 61;161-265.

2. Koffka K. Principles of Gestalt psychology. New York: Harcourt; 1935. 720 p.

3. Groen IIA, Silson EH, Baker $\mathrm{Cl}$. Contributions of low- and high-level properties to neural processing of visual scene sin the human brain. Philos Trans R Soc Lond B Biol Sci. 2017;372(1714).

4. Field DJ, Hayes A, Hess RF. Contour integration by the human visual system: evidence for a local “association field". Vision Research. 1993;33(2):173-93.

5. Hess R, Field D. Integration of contours: New insights. Trends in Cognitive Sciences. 1999;3(12):480-6.

6. Neri P, Morrone MC, Burr DC. Seeing biological motion. Nature. 1998;395:894-6.

7. Frégnac $Y$, Bathellier B. Cortical correlates of low-level perception: from neural circuits to percepts. Neuron. 2015;88(1):110-26.

8. Felleman DJ, Van Essen DC. Distributed hierarchical processing in the primate cerebral cortex. Cerebral Cortex. 1991;1(1):1-47.

9. Heeger DJ, Simoncelli EP, Movshon JA. Computational models of cortical visual processing. Proceedings Natlonal Academy of Science USA. 1996;93:623-7.

10. Serre T, Oliva A, Poggio T. A feedforward architecture accounts for rapid categorization. Proceedings Natlonal Academy of Sciences of the USA. 2007;104(15):6424-9.

11. Li W, Piëch V, Gilbert CD. Contour saliency in primary visual cortex. Neuron. 2006;50:951-62.

12. Polat U, Mizobe K, Pettet MW, Kasamatsu T, Norcia AM. Collinear stimuli regulate visual responses depending on cell's contrast threshold. Nature. 1998;391:580-4.

13. Mizobe K, Polat U, Pettet MW, Kasamatsu T. Facilitation and suppression of single striate-cell activity by spatially discrete pattern stimuli presented beyond the receptive field. Visual Neuroscience. 2001;18:377-91.

14. Kasamatsu T, Miller R, Zhu Z, Chang M, Ishida Y. Collinear facilitation is independent of receptive-field expansion at low contrast. Experimental Brain Research. 2010;201(3):453-65.

15. Li W, Piëch V, Gilbert CD. Learning to link visual contours. Neuron. 2008;57:442-51.

16. Gilbert CD, Li W. Top-down influences on visual processing. National Review of Neuroscience. 2013;14(5):350-63.

17. Movshon A, Adelson EH, Gizzi MS, Newsome WT. The analysis of moving visual patterns. In: Chagas C, Gattass, R.,Gross, C., editor. Pattern Recognition Mechanisms: Pontificiae Academiae Scientiarum Scripta Varia. 1985;54:117-51.

18. Castelo-Branco M, Formisano E, Backes W, Zanella F, Neuenschwander S, Singer W, et al. Activity patterns in human motion-sensitive areas depend on the interpretation of global motion. Proceedings of the Natlonal Academy of Science U S A. 2002;99:13914-9.

19. Aaen-Stockdale C, Thompson B. Visual Motion: From Cortex to Percept. IntechOpen. 2011. 
20. Aissani C, Cottereau B, Dumas G, Paradis A-L, Lorenceau J. Magnetoencephalographic signatures of visual form and motion binding. Brain Research. 2011;1408:27-41.

21. Hirsch JA, Alonso JM, Reid RC, Martinez LM. Synaptic integration in striate cortical simple cells. The Journal of Neuroscience,. 1998;18:9517-28.

22. Ferster D, Miller KD. Neural mechanisms of orientation selectivity in the visual cortex. Annual Review of Neuroscience. 2000;23:441-71.

23. Douglas RJ, Martin KA. Neuronal circuits of the neocortex. Annual Review of Neuroscience. 2004;27:41951.

24. Priebe NJ, Ferster D. Mechanisms of neuronal computation in mammalian visual cortex. Neuron. 2012;75(2):194-208.

25. Frégnac $Y$, Bringuier $V$. Spatio-temporal dynamics of synaptic integration in cat visual cortical receptive fields. In: Braitenberg AAaV, editor. Brain Theory: Biological basis and computational theory of Vision. 1996. p. 143-99.

26. Bringuier V, Chavane F, Glaeser L, Fregnac Y. Horizontal propagation of visual activity in the synaptic integration field of area 17 neurons. Science. 1999;283:695-9.

27. Baudot P. Nature is the code: high temporal precision and low noise in V1. Paris: University Paris VI; 2006.

28. Haider B, Krause MR, Duque A, Yu Y, Touryan J, Mazer JA, et al. Synaptic and network mechanisms of sparse and reliable visual cortical activity during nonclassical receptive field stimulation. Neuron. 2010;65(1):107-21.

29. Gerard-Mercier F, Carelli PV, Pananceau M, Troncoso XG, Fregnac Y. Synaptic Correlates of Low-Level Perception in V1. The Journal of Neuroscience. 2016;36(14):3925-42.

30. Ozeki H, Finn IM, Schaffer ES, Miller KD, Ferster D. Inhibitory Stabilization of the Cortical Network Underlies Visual Surround Suppression. Neuron. 2016;62(4):578-92.

31. Das A, Gilbert CD. Long-range horizontal connections and their role in cortical reorganization revealed by optical recording of cat primary visual cortex. Nature. 1995;375(6534):780-4.

32. Grinvald A, Lieke EE, Frostig RD, Hildesheim R. Cortical point-spread function and long-range lateral interactions revealed by real-time optical imaging of macaque monkey primary visual cortex. The Journal of Neuroscience. 1994;14:2545-68.

33. Slovin $\mathrm{H}$, Arieli A, Hildesheim R, Grinvald A. Long-term voltage-sensitive dye imaging reveals cortical dynamics in behaving monkeys. Journal of Neurophysiology. 2002;88:3421-38.

34. Reynaud A, Masson GS, Chavane F. Dynamics of Local Input Normalization Result from Balanced Shortand Long-Range Intracortical Interactions in Area V1. The Journal of Neuroscience. 2012;32:12558-69.

35. Muller L, Reynaud A, Chavane F, Destexhe A. The stimulus-evoked population response in visual cortex of awake monkey is a propagating wave. Nature 2014;5:3675.

36. Davis ZW, Muller L, Martinez-Trujillo J, Sejnowski T, Reynolds JH. Spontaneous travelling cortical waves gate perception in behaving primates. Nature. 2020;587(7834):432-6.

37. Benucci A, Frazor RA, Carandini M. Standing waves and traveling waves distinguish two circuits in visual cortex. Neuron. 2007;55(1):103-17. 
38. Sato TK, Nauhaus I, Carandini M. Traveling waves in visual cortex. Neuron. 2012;2:218-29.

39. Jancke D, Erlhagen W, SchÜner G, Dinse HR. Shorter latencies for motion trajectories than for flashes in population responses of cat primary visual cortex. Journal of Physiology. 2004;556(3):971-82.

40. Chavane F, Sharon D, Jancke D, Marre O, Fregnac Y, Grinvald A. Lateral Spread of Orientation Selectivity in V1 is Controlled by Intracortical Cooperativity. Frontiers System Neuroscience. 2011;5:4.

41. $\mathrm{Xu} \mathrm{W,} \mathrm{Huang} \mathrm{X}$, Takagaki $\mathrm{K}, \mathrm{Wu}$ J. Compression and reflection of visually evoked cortical waves. Neuron. 2007;55:119-29.

42. Polat U, Sagi D. Lateral interactions between spatial channels, suppression and facilitation revealed by lateral masking experiments. Vision Research. 1993;33:993-9.

43. Fournier J, Monier C, Pananceau M, Frégnac Y. Adaptation of the Simple or Complex nature of V1 receptive fields to visual statistics. Nature Neuroscience. 2011;14:1053-60.

44. Fournier Y, Monier C, Levy M, Marre O, Sari K, Kisvarday ZF, et al. Hidden Complexity of synaptic receptive fields in cat primary visual cortex. The Journal of Neuroscience. 2014;34(16):5515-28.

45. Chavane F. Diversité d'expression fonctionnelle du champ d'intégration synaptique : une re-évaluation intracellulaire des champs récepteurs des neurones du cortex visuel primaire. Paris: University Paris VI; 1999.

46. Hubel DH, Wiesel TN. Receptive fields, binocular interaction and functional architecture in the cat's visual cortex. Journal of Physiology (London). 1962;160:106-54.

47. Series P, Georges S, Lorenceau J, Fregnac Y. Orientation dependent modulation of apparent speed: a model based on the dynamics of feed-forward and horizontal connectivity in V1 cortex. Vision Research. 2002;42(25):2781-97.

48. Nelson JI, Frost BJ. Intracortical facilitation among co-oriented co-axially aligned simple cells in cat striate cortex. Experimental Brain Research. 1985;61:54-61.

49. Li C, Li W. Extensive integration field beyond the classical receptive field of cat's striate cortical neurons: Classification and tuning properties. Vision Research. 1994;34:2337-55.

50. Kapadia MK, Ito M, Gilbert CD, Westheimer G. Improvement in visual sensitivity by changes in local context: Parallel studies in human observers and in V1 of alert monkeys. Neuron. 1995;15:843-56.

51. Sengpiel F, Sen A, Blakemore C. Characteristics of surround inhibition in cat area 17. Experimental Brain Research. 1997;116:216-28.

52. Walker GA, Ohzawa I, Freeman RD. Suppression outside the classical cortical receptive field. Visual Neuroscience. 2000;17:369-79.

53. Chen $\mathrm{K}$, Song $\mathrm{XM}$, Li CY. Contrast-dependent variations in the excitatory classical receptive field and suppressive nonclassical receptive field of cat primary visual cortex. Cerebral Cortex. 2013;23(23):28392.

54. Gilbert CD, Wiesel TN. Columnar specificity of intrinsic horizontal and corticocortical connections in cat visual corte. The Journal of Neuroscience. 1989;9:2432-42.

55. Kisvarday ZF, Toth E, Rausch M, Eysel UT. Orientation-specific relationship between populations of excitatory and inhibitory lateral connections in the visual cortex of the cat. Cerebral cortex. 1997;7(7):605-18. 
56. Schmidt KE, Goebel R, Löwell S, Singer W. The perceptual grouping criterion of colinearity is reflected by anisotropies of connections in the primary visual cortex. European Journal of Neuroscience. 1997;9:1083-9.

57. Fitzpatrick D. The functional organization of local circuits in visual cortex: insights from the study of tree shrew striate cortex. Cerebral Cortex. 1996;6:329-41.

58. Chisum J, Fitzpatrick D. The contribution of vertical and horizontal connections to the receptive field center and surround in V1. Neural Networks. 2004;17:681-93.

59. Bosking $\mathrm{WH}$, Zhang $\mathrm{Y}$, Chofield $\mathrm{B}$, Fitzpatrick D. Orientation selectivity and the arrangement of horizontal connections in tree shrew striate corte. The Journal of Neuroscience. 1997;17(6):2112-27.

60. McGuire BA, Gilbert CD, K. RP, Wiesel TN. Targets of horizontal connections in macaque primary visual cortex. Journal of Comparative Neurology. 1991;305:370-92.

61. Malach R, Amir $Y$, Harel M, Grinvald A. Relationship between intrinsic connections and functional architecture revealed by optical imaging and in vivo targeted biocytin injections in primate striate cortex. Proceedings of the Natlonal Academy of Sciences of the USA. 1993;90:10469-73.

62. Sincish LC, Blasdel GG. Oriented axon projections in primary visual cortex of the monkey. The Journal of Neuroscience. 2001;21:4416.

63. Kapadia MK, Westheimer G, Gilbert CD. Spatial distribution of contextual interactions in primary visual cortex and in visual perception. Journal of Neurophysiology. 2000;84:2048-62.

64. Pettigrew JD, Nikara T, Bishop PO. Binocular interaction on single units in cat striate cortex: simultaneous stimulation by single moving slit with receptive fields in correspondence. Experimental Brain Research. 1968;6:391-410.

65. Orban GA, Kennedy $\mathrm{H}$, Maes $\mathrm{H}$. Response to movement of neurons in areas 17 and 18 of the cat: velocity sensitivity. Journal of Neurophysiology. 1981;45:1043-58.

66. Hammond P, Smith AT. On the sensitivity of complex cells in feline striate cortex to relative motion. Experimental Brain Research. 1982;47(3):457-60.

67. Duysens J, Orban JA, Cremieux J. Functional basis for the preference for slow movement in area 17 of the cat. Vision Research. 1984;24(1):17-24.

68. Orban GA. Neuronal operations in the visual cortex. Studies in brain function. Berlin: Springer-Verlag; 1984.

69. Albright TD. Direction and Orientation Selectivity of Neurons in Visual Area MT of the Macaque. Brain Research. 1984;1408:27-40.

70. Geisler WS. Motion streaks provide a spatial code for motion direction. Nature. 1999;400(6739):65-9.

71. Geisler WS, Albrecht DG, Crane A, Stern L. Motion direction signals in the primary visual cortex of cat and monkey. Visual Neuroscience. 2001;18:5001-516.

72. Frazor RD, Albrecht DG, Geisler WS, Crane AM. Visual cortex neurons of monkeys and cats: Temporal dynamics of the spatial frequency response functio. Journal of Neurophysiology. 2004;91:2607-27.

73. Crook JM, Worgötter F, Eysel UT. Velocity invariance of preferred axis of motion for single spot stimuli in simple cells of cat striate cortex. Experimental Brain Research. 1994;102:175-80. 
74. Basole A, White LE, Fitzpatrick D. Mapping multiple stimulus features in the population response of visual cortex. Nature. 2003;423:775-87.

75. An X, Gong H, McLoughlin N, Yang Y, Wang W. The mechanism for processing random-dot motion at various speeds in early visual cortices. PLoS ONE. 2014;9(3):e93115.

76. Georges S, Series P, Fregnac Y, Lorenceau J. Orientation dependent modulation of apparent speed: psychophysical evidence. Vision Research. 2002;42:2757-72.

77. Mumford DB. On the computational architecture of the neocortex II: The role of cortico-cortical loops. Biological Cybernetics. 1992;66:241-51.

78. Burr DC, Morgan MJ. Motion deblurring in human vision. Proceedings of the Royal Society London. 1997;264:431-6.

79. Zhou H, Friedman HS, Von der Heydt R. Coding of border ownership in monkey visual cortex. The Journal of Neuroscience. 2000;20:6594-611.

80. Born RT, Bradley DC. Structure and Function of Visual Area MT. Annual Review of Neuroscience. 2005;28:157-89.

81. Roe AW, Chelazzi L, Connor CE, Conway B, Fujita C, Gallant JL, et al. Toward a Unified Theory of Visual Area V4. Neuron. 2012;74(1):12-29.

82. Schwabe L, Ichida JM, Shushruth S, Mangapathy P, Angelucci A. Contrast-dependence of surround suppression in Macaque VI: experimental testing of a recurrent network model. Neuroimage. 2010;52:777-92.

83. Poort J, Raudies F, Wannig A, Lamme VA, Neumann H, Roelfsema PR. The role of attention in figureground segregation in areas V1 and V4 of the visual cortex. Neuron. 2012;75(1):143-56.

84. Nassi JJ, Lomber SG, Born RT. Corticocortical feedback contributes to surround suppression in V1 of the alert primate. The Journal of Neuroscience. 2013;33:8504-17.

85. Bastos AM, Vezoli J, Bosman CA, Schoffelen JM, Oostenveld R, Dowdall JR, et al. Visual areas exert feedforward and feedback influences through distinct frequency channels. Neuron. 2015;85(2):390401.

86. Lamme VA, Supèr $H$, Landman R, Roelfsema PR, Spekreijse $H$. The role of primary visual cortex (V1) in visual awareness. Vision Research. 2000;40(10-12):1507-21.

87. Angelucci A, Levitt JB, Walton EJ, Hupé JM, Bullier J, Lund JS. Circuits for local and global signal integration in primary visual cortex. J Neurosci. 2002;22:8633-46.

88. Shushruth S, Ichida JM, Levitt JB, Angelucci A. Comparison of spatial summation properties of neurons in macaque V1 and V2. Journal of Neurophysiology. 2009;102(4):2069-83.

89. Nurminen L, Angelucci A. Multiple components of surround modulation in primary visual cortex: multiple neural circuits with multiple functions? . Vision Research. 2014;104:47-56.

90. Angelucci A, Bijanzadeh M, Nurminen L, Federer F, Merlin S, Bressloff PC. Circuits and Mechanisms for Surround Modulation in Visual Corte. Annual Review of Neuroscience. 2017;40:425-51.

91. Roland PE, Hanazawa A, Undeman C, .Eriksson D, Tompa T, Nakamura H, et al. Cortical feedback depolarization waves: $A$ mechanism of top-down influence on early visual areas. Proceedings of the National Academy of Sciences of the USA. 2006;103(33):12586-91. 
92. Callaway EM, Katz LC. Emergence and refinement of clustered horizontal connections in cat striate cortex. The Journal of Neuroscience. 1990;10(4):1134-53.

93. Angelucci A, Bressloff PC. The contribution of feedforward, lateral and feedback connections to the classical receptive field center and extra-classical receptive field surround of primate V1 neurons. Progress in Brain Research. 2006;154:93-121.

94. Polat U, Sagi D. The architecture of perceptual spatial interactions. Vision Research. 1994;34:73-8.

95. Polat U, Sagi D. Spatial interactions in human vision, from near to far via experience-dependent cascades of connections. Proceeding of the National Academy of Sciences of the USA. 1994;91:1206-9.

96. Polat U, Tyler CW. What pattern the eye sees best. Vision Research. 1999;39(5):887-95.

97. Bullier J, Hupe JM, James AC, Girard P. The role of feedback connections in shaping the responses of visual cortical neurons. Progress in Brain Research. 2001;134:193-204.

98. Friston KJ, Parr T, de Vries B. The graphical brain: belief propagation and active inference. Network Neuroscience. 2017;1(4):381-414.

99. Benvenuti G, Chemla S, Boonman A, Perrinet L, Masson G, Chavane F. Anticipatory responses along motion trajectories in awake monkey area V1. 2020. bioRxiv 2020.03.26.010017.

100. Tanifuji M, Sugiyama T, Murase K. Horizontal propagation of excitation in rat visual cortical slices revealed by optical imaging. Science. 1994;266(5187):1057-9.

101. Gilad A, Meirovithz E, Leshem A, Arieli A, Slovin H. Collinear stimuli induce local and cross-areal coherence in the visual cortex of behaving monkeys. PLoS ONE. 2012;7(11):e49391.

102. Frégnac Y. Reading out the synaptic echoes of low-level perception in V1. Lecture Notes in Computer Science. 2012;7583:486-95.

103. Rao RPN, Ballard DH. Predictive Coding in the Visual Cortex: A Functional Interpretation of Some ExtraClassical Receptive Field Effects. Nature Neuroscience. 1999;2:79-87.

104. Martin AB, Von der Heydt R. Spike synchrony reveals emergence of proto-objects in visual corte. The Journal of Neuroscience. 2015;35:6860-70.

105. Kaplan BA, Lansner A, Masson GS, Perrinet LU. Anisotropic connectivity implements motion-based prediction in a spiking neural network. Frontiers in Computational Neuroscience. 2013;7:112

106. Cowey A, Rolls ET. Human cortical magnification factor and its relation to visual acuity. Experimental Brain Research. 1974;21:447-54.

107. Tusa RJ, Palmer LA, Rosenquist AC. The retinotopic organization of area 17 (striate cortex) in the cat. Journal of Comparative Neurology. 1978;177(2):213-35.

108. Hirsch JA, Gilbert CD. Synaptic physiology of horizontal connections in the cat's visual cortex. The Journal of Neuroscience. 1991;11(6):1800-9.

109. Deco G, Roland P. The role of multi-area interactions for the computation of apparent motion. Neuroimage. 2010;51:1018-26.

110. Veltz R. Interplay between synaptic delays and propagation delays in neural field equation. SIAM Journal on Applied Dynamical Systems. 2013;12(3):1566-612. 
111. Chisum HJ, Mooser F, Fitzpatrick D. Emergent properties of layer 2/3 neurons reflect the collinear arrangement of horizontal connections in tree shrew visual corte. The Journal of Neuroscience. $2003 ; 23(7): 2947-60$.

112. Muckli L, Kohler A, Kriegeskorte N, Singe rW. Primary visual cortex activity along the apparent-motion trace reflects illusory perception. PLOS Biology. 2005;3(8):e265.

113. Frégnac Y. The "dynamic association field" of visual cortex neurons: a fit between natural perception and eye-movements? . American Society for Neuroscience Symposium "Mechanisms and Functions of Visual Signals from Beyond the Classical Receptive Field in Primary Visual Cortex"; San Diego2007.

114. Frégnac Y. Searching for a fit between the silent surround of V1 receptive fields and eye-movements. Journal of Vision. 2013;13(9):1380.

115. Tatler BW, Wade NJ, Kwan H, Findlay JM, Velichkovsky BM. Yarbus, eye movements, and vision. Iperception. 2010;1(1):7-27. 


\section{Abbreviations}

1406. AM: Apparent motion

1407. ASHP: Apparent Speed of Horizontal Propagation

1408. CP: Centripetal

1409. CF: Centrifugal

1410. CROSS-axis : cross-oriented with the motion axis

1411. CROSS-RF : cross-oriented (orthogonal) with the RF orientation preference

1412. FF: Feedforward

1413. GP: Gabor Patch

1414. DN: dense noise

1415. ISO-axis: collinear or parallel to the motion axis

1416. ISO-RF: collinear or parallel to the RF orientation preference

1417. MDF: Minimal Discharge Field

1418. NHP: Non-Human Primate

1419. RF: Receptive Field

1420. RND: Randomized

1421. SRF: Subthreshold synaptic Receptive Field

1422. SN: sparse noise

1423. V1: Area 17 in the cat, Primary Visual Cortex

\section{Conflict of Interest Statement :}

1425. The authors declare no competing financial interests. 


\section{Funding :}

1427. This work was performed at the UNIC (Unit of Neuroscience, Information and Complexity), CNRS, UPR

1428. CNRS 3293, Gif-sur-Yvette, 91198 France. It was supported by the Centre National de la Recherche

1429. Scientifique (CNRS) and grants to Y.F. from the Agence Nationale de la Recherche (ANR-Horizontal-V1

1430. (ANR-17-CE37-0006), the Paris-Saclay IDEX (NeuroSaclay and i-Code) and the FET-Proactive Grant

1431. Agreement No 269921 (BrainScales). This project has also received postdoctoral fellowship under the

1432. Marie Skłodowska-Curie grant agreements No 659593 (Proaction Perception) and No 302215 (Brain

1433. Percepts). B.L. and Y. P. were supported by The Human Brain Project and the ANR-Horizontal-V1.

1434.

\section{Author contributions :}

1436. Y.F. conceived of the study.

1437. Y.F., P.B., X.T., B.LB., C.D., M.P., Y.P. and C.M. designed the experiments.

1438. P.B., M.P., X.T., C.D. and B.LB. collected the intracellular data.

1439. B.LB., C.D., X.T. ("radial” protocol) and P.B. ("cardinal” protocol) implemented the data analysis.

1440. C.D. and M.P. designed the figures.

1441. Y.F. and B. LB. wrote the manuscript. 


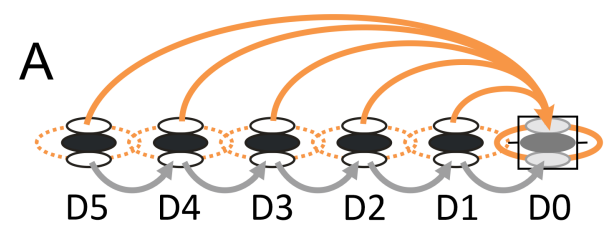

B
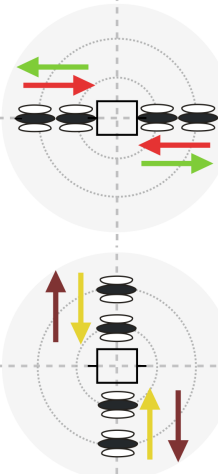

antan
C SECTOR
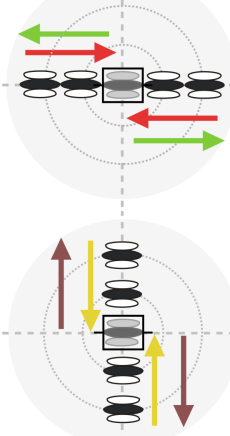

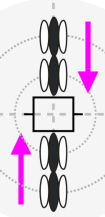

FULL
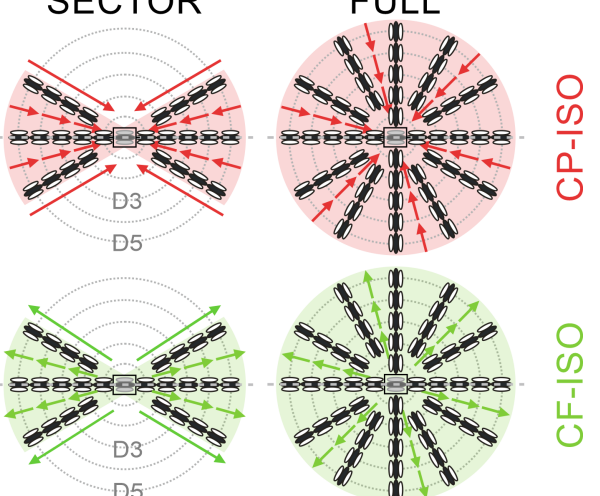

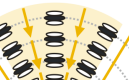

훙- D3-D5

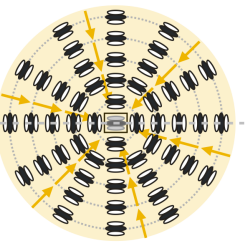

$\infty$
$\infty$
0
$\propto$
0
0
0
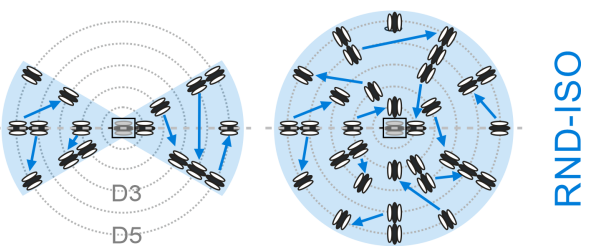
A

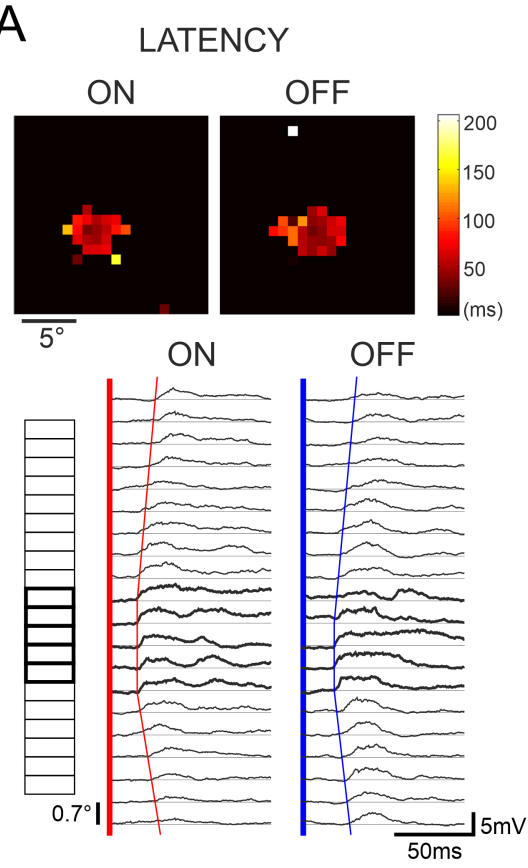

B
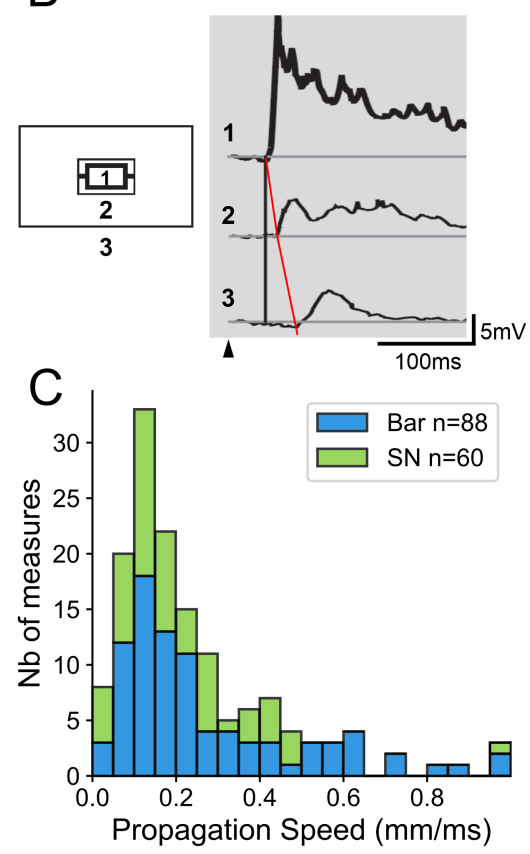


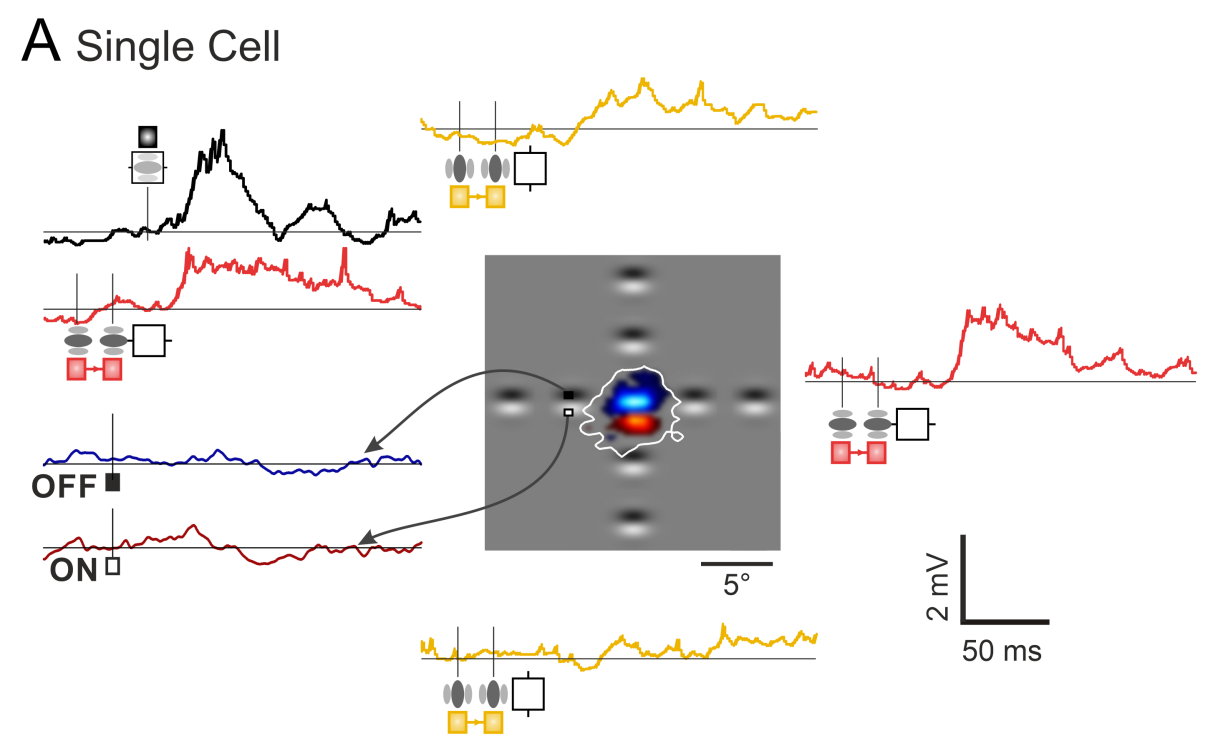

B Population ( $n=13)$
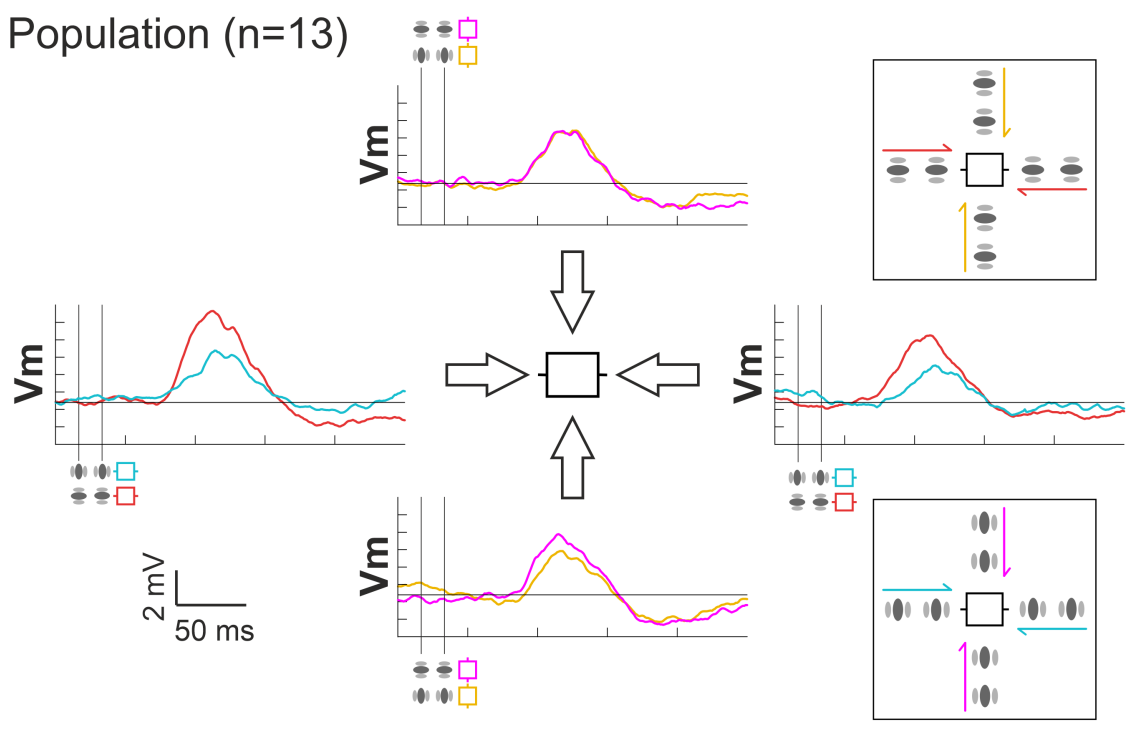

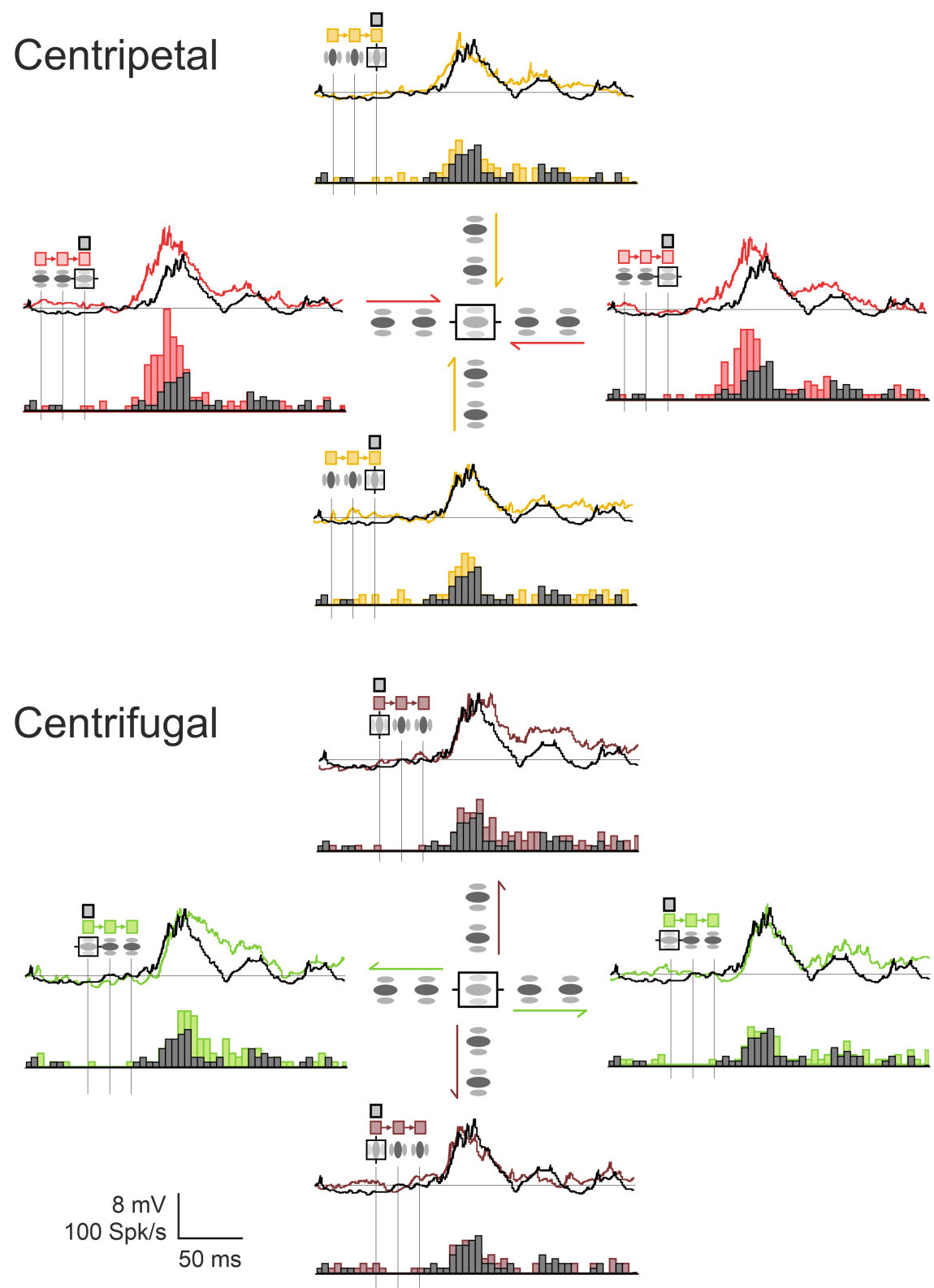
bioRxiv preprint doi: https://doi.org/10.1101/2021.09.10.459746; this version posted September 11, 2021. The copyright holder for this preprint (which was not certified by peer review) is the author/funder. All rights reserved. No reuse allowed without permission.

A
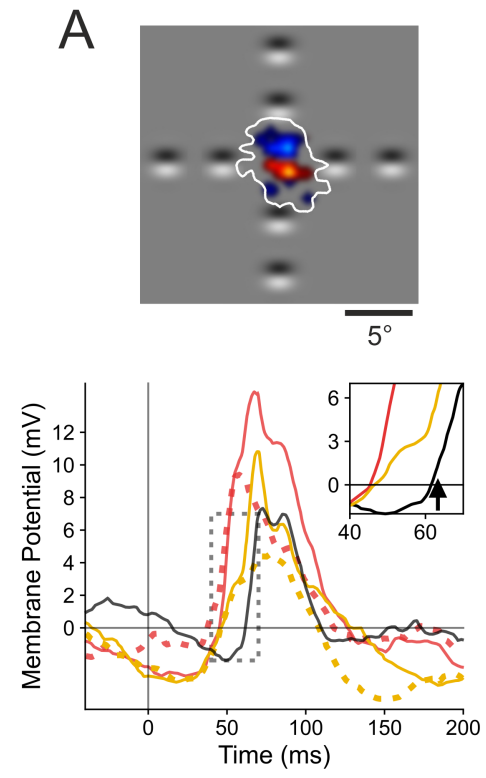

B
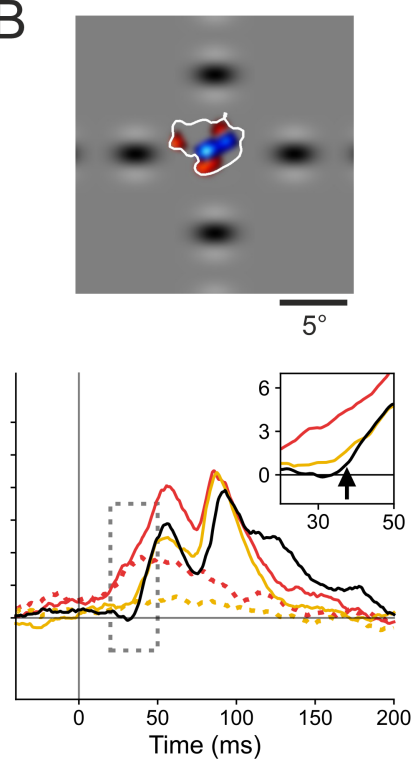
Single Cell

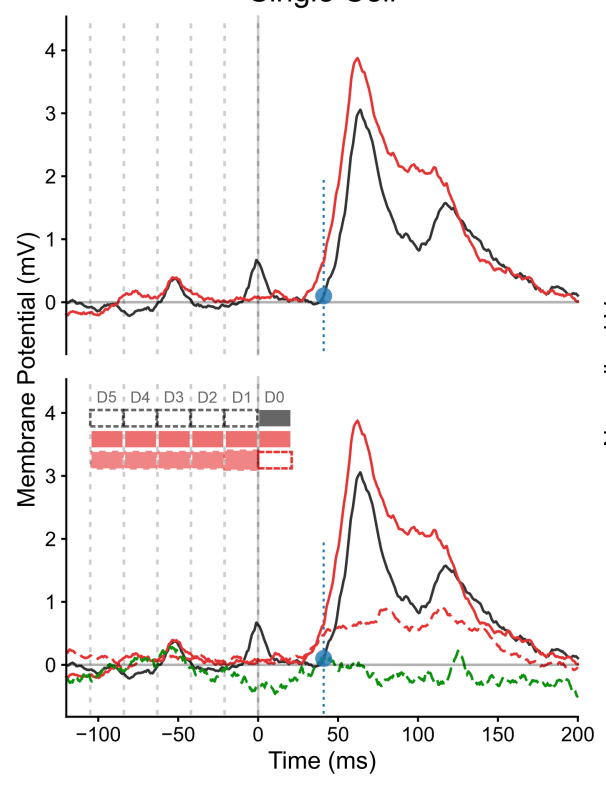

Population Average

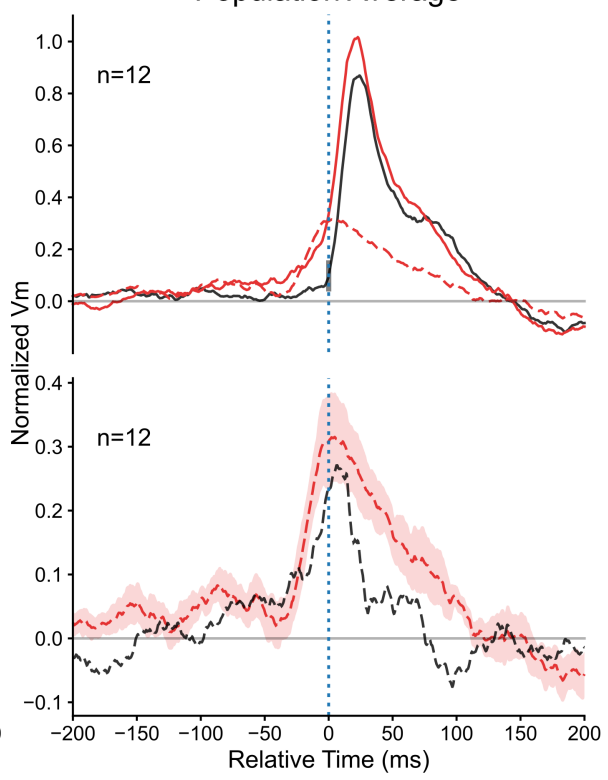



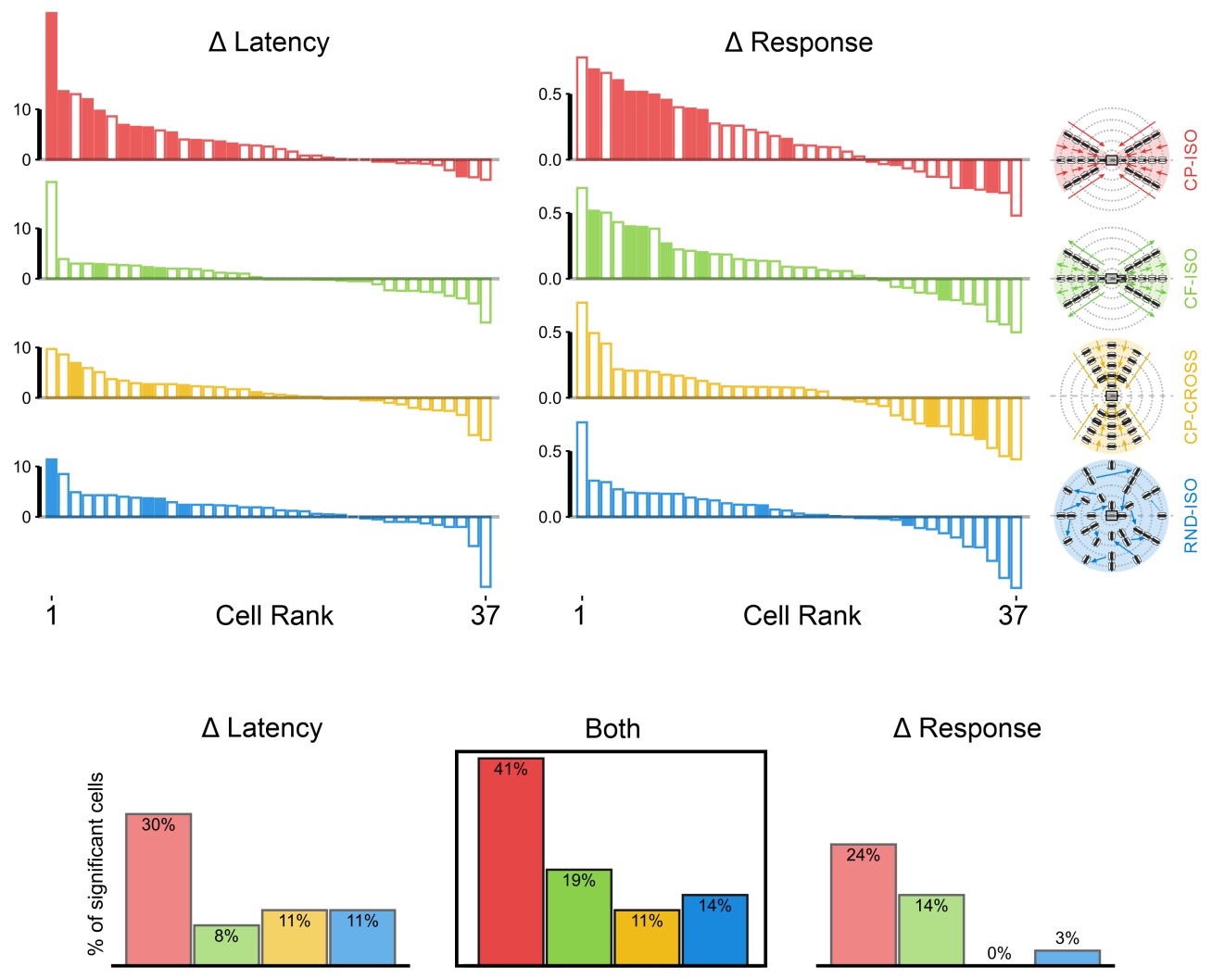
Single Cell
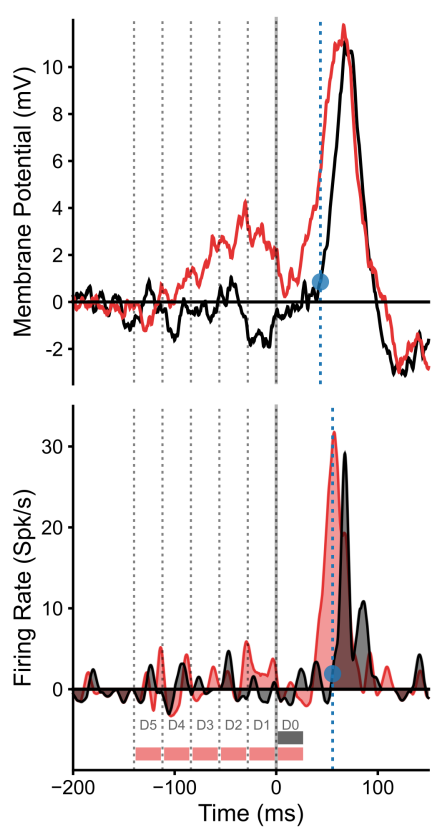

Population Average
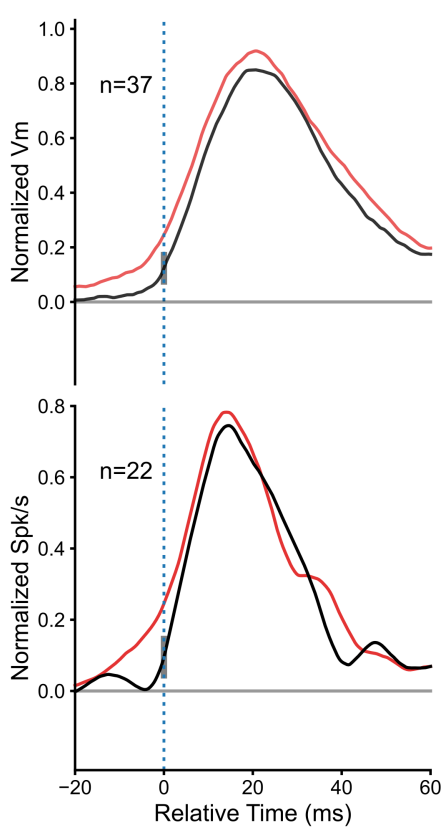

Significant Cells
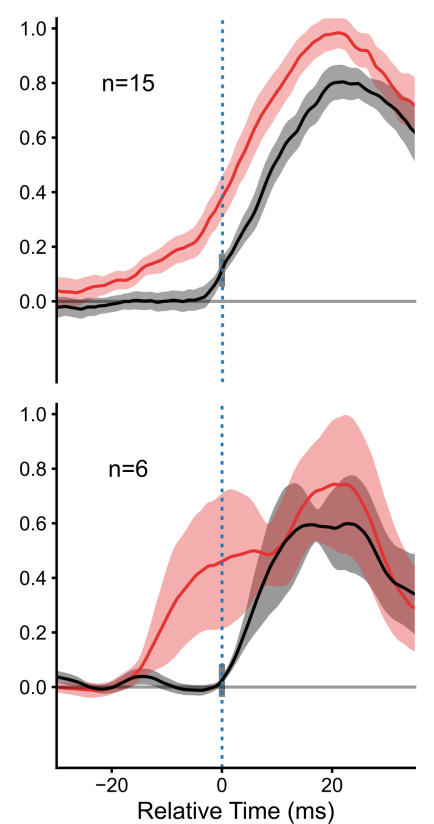

Significant Cells

(all conditions)
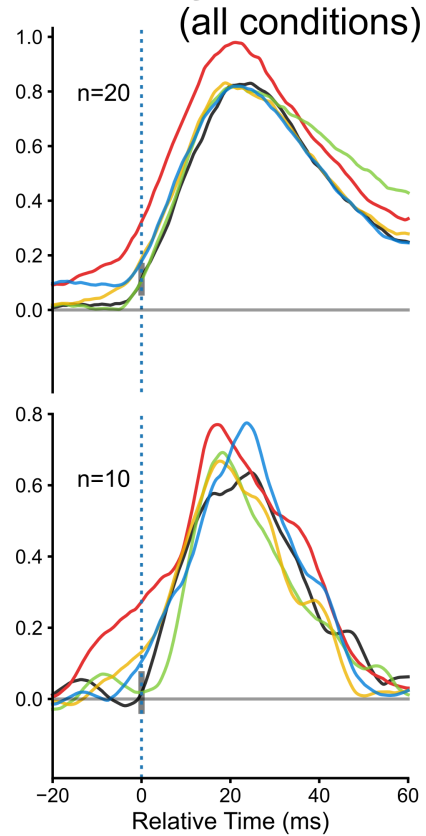

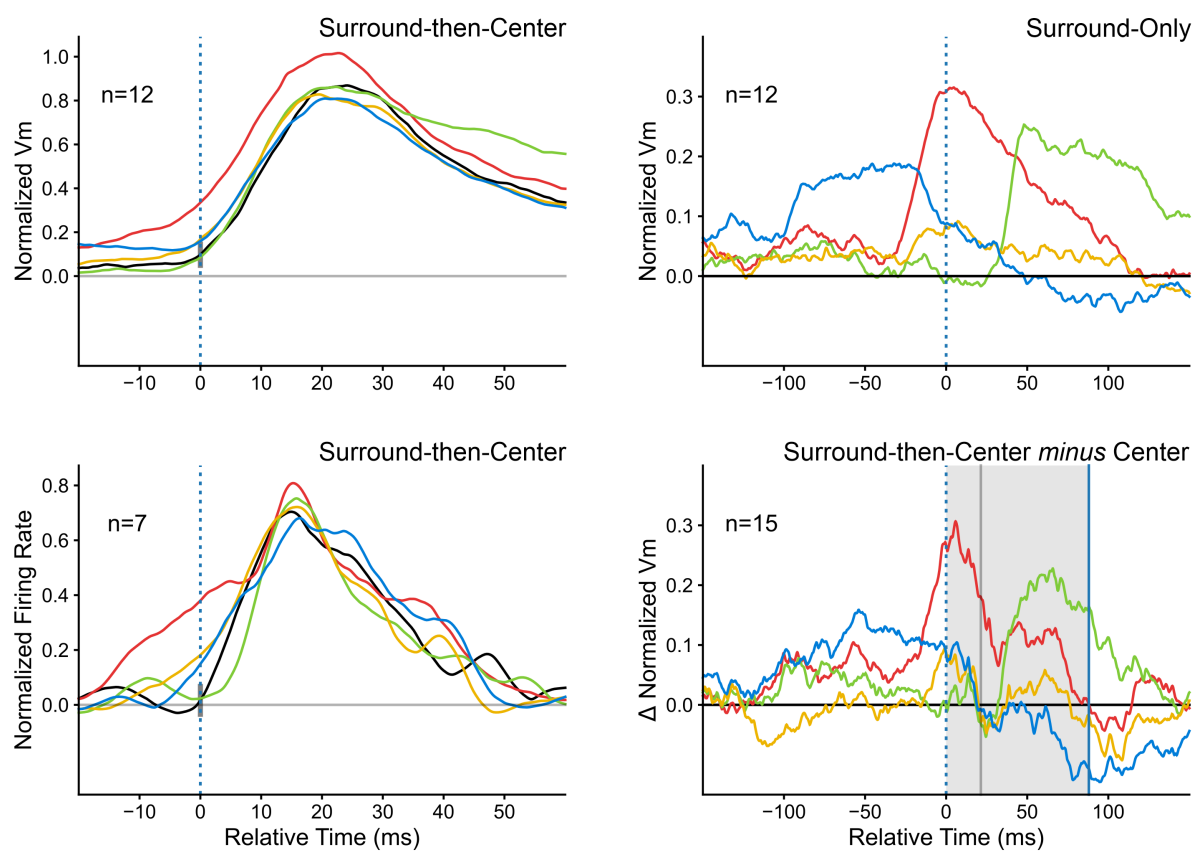

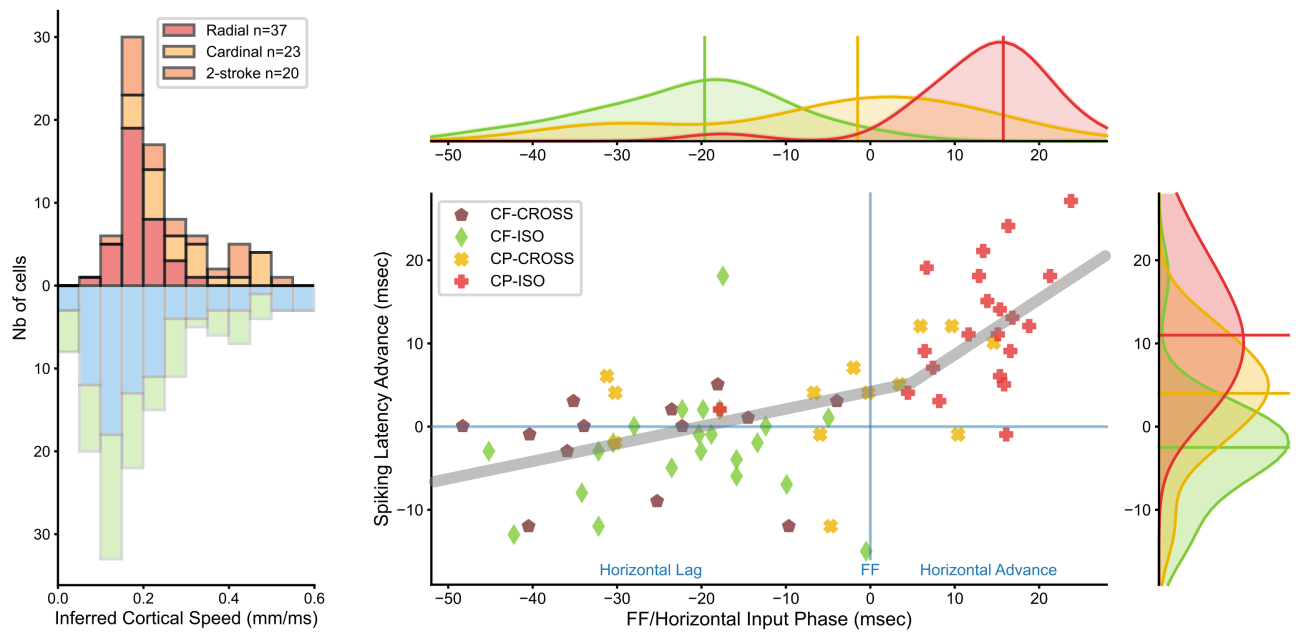

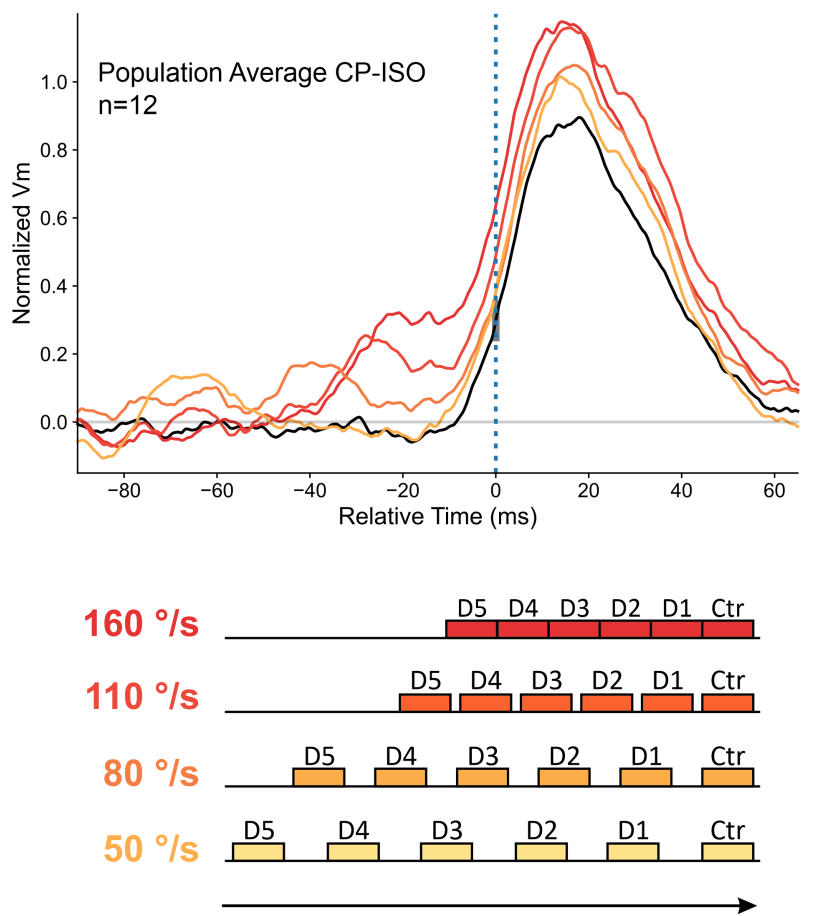

Time 